\title{
Optical second harmonic generation in the centrosymmetric magnetic semiconductors EuTe and EuSe
}

\author{
B. Kaminski, ${ }^{1}$ M. Lafrentz, ${ }^{1}$ R. V. Pisarev, ${ }^{2}$ D. R. Yakovlev, ${ }^{1,2}$ V. V. Pavlov, ${ }^{2}$ V. A. Lukoshkin, ${ }^{2}$ A. B. Henriques, ${ }^{3}$ \\ G. Springholz, ${ }^{4}$ G. Bauer, ${ }^{4}$ E. Abramof, ${ }^{5}$ P. H. O. Rappl, ${ }^{5}$ and M. Bayer ${ }^{1}$ \\ ${ }^{1}$ Experimentelle Physik 2, Technische Universität Dortmund, D-44221 Dortmund, Germany \\ ${ }^{2}$ Ioffe Physical-Technical Institute, Russian Academy of Sciences, 194021 St. Petersburg, Russia \\ ${ }^{3}$ Instituto de Física, Universidade de São Paulo, 05315-970 São Paulo, SP, Brazil \\ ${ }^{4}$ Institut für Halbleiter- und Festkörperphysik, Johannes Kepler Universität Linz, 4040 Linz, Austria \\ ${ }^{5}$ LAS-INPE, 12227-010 São José dos Campos, SP, Brazil
}

(Received 13 January 2010; revised manuscript received 5 March 2010; published 5 April 2010)

\begin{abstract}
The magnetic europium chalcogenide semiconductors EuTe and EuSe are investigated by the spectroscopy of second harmonic generation (SHG) in the vicinity of the optical band gap formed by transitions involving the $4 f$ and $5 d$ electronic orbitals of the magnetic $\mathrm{Eu}^{2+}$ ions. In these materials with centrosymmetric crystal lattice the electric-dipole SHG process is symmetry forbidden so that no signal is observed in zero magnetic field. Signal appears, however, in applied magnetic field with the SHG intensity being proportional to the square of magnetization. The magnetic field and temperature dependencies of the induced SHG allow us to introduce a type of nonlinear optical susceptibility determined by the magnetic-dipole contribution in combination with a spontaneous or induced magnetization. The experimental results can be described qualitatively by a phenomenological model based on a symmetry analysis and are in good quantitative agreement with microscopic model calculations accounting for details of the electronic energy and spin structure.
\end{abstract}

DOI: 10.1103/PhysRevB.81.155201

PACS number(s): 75.50.Pp, 42.65.Ky, 78.20.Ls

\section{INTRODUCTION}

Nonlinear optics is a highly active field of solid-state physics with important implementations in modern technology. Within the vast area of nonlinear optics the processes of harmonics generation, sum and difference frequency generation, and parametric scattering play a particularly important role. ${ }^{1,2}$ Harmonics generation is associated with higher order optical susceptibilities, and it gives access to the microscopic mechanisms of nonlinear light-matter interaction and unique information about the crystallographic, electronic and magnetic structure, which is inaccessible by linear optics techniques. ${ }^{1-3}$

Second harmonic generation (SHG) has attracted considerable interest in fundamental physics because of its exceptional sensitivity to space and time symmetry violations. ${ }^{3}$ Another important feature of SHG is that it gives specific information in studies of surfaces and interfaces. ${ }^{4-8}$ The various technological applications of SHG are based on materials with high second order nonlinear susceptibility which allow also fulfillment of the condition of phase matching.

In the most general case of the multipole approximation, the contributions to the three-photon SHG process, taking into account no more than one second-order transition as well as the indistinguishability of the two incident photons, can be written in matrix form as ${ }^{9,10}$

$$
\left(\begin{array}{c}
\mathbf{P}^{2 \omega} \\
\mathbf{M}^{2 \omega} \\
\hat{Q}^{2 \omega}
\end{array}\right) \propto\left(\begin{array}{ccc}
\chi^{e e e} & \chi^{e e m} & \chi^{e e q} \\
\chi^{\text {mee }} & 0 & 0 \\
\chi^{q e e} & 0 & 0
\end{array}\right)\left(\begin{array}{c}
\mathbf{E}^{\omega} \mathbf{E}^{\omega} \\
\mathbf{E}^{\omega} \mathbf{B}^{\omega} \\
\mathbf{E}^{\omega} \hat{Q}^{\omega}
\end{array}\right),
$$

where $\mathbf{P}^{2 \omega}, \mathbf{M}^{2 \omega}$, and $\hat{Q}^{2 \omega}$ denote electric polarization, magnetization, and electric-quadrupole polarization, respectively, at the SHG frequency, $2 \omega . \mathbf{E}^{\omega}, \mathbf{B}^{\omega}$ and $\hat{Q}^{\omega}$ are the electric, magnetic and electric-quadrupole fields, respectively, at the fundamental frequency, $\omega$. The five independent nonlinear susceptibilities $\chi^{k l n}$ describe various types of electric dipole (ED), magnetic dipole (MD), and electric-quadrupole (EQ) processes contributing to coherent SHG. They depend on the crystallographic, electronic and magnetic structure, and on the types of nonlinear processes involved in the light-matter interaction.

The strongest interaction is given by the ED susceptibility $\chi^{e e e}$, which is only allowed in noncentrosymmetric crystal media due to the parity selection rule. ${ }^{1,2}$ Modern technology allows fabrication of controlled artificial structures, in which an inversion center is either present or not, which are of high interest for nonlinear optical studies and new applications. In addition, the ED susceptibility plays a dominant role in centrosymmetric media at surfaces or interfaces where the inversion symmetry is broken. ${ }^{4-6}$ Here, one should note that the analysis of experimental SHG data from surfaces and interfaces in centrosymmetric media must be carried out with great care because, in general, EQ and MD bulk effects can overlap with ED surface/interface effects. ${ }^{11,12}$

Up to now, most of the fundamental and applied SHG research was devoted to the $\chi^{e e e} \mathrm{ED}$ nonlinear susceptibility which is inherent to piezoelectric and ferroelectric materials used in second, third, and higher order frequency multipliers, sum and difference frequency generators, optical parametric oscillators, etc. A crucial condition for practical applications of these materials is the possibility to fulfill the phasematching requirements between the fundamental and second, or higher order harmonics.

The other $\chi^{k l n}$ susceptibilities in Eq. (1) involve simultaneous couplings of $\mathrm{ED}, \mathrm{MD}$, or $\mathrm{EQ}$ processes and, as a rule, are several orders of magnitude smaller than $\chi^{e e e}$. Nevertheless, in centrosymmetric media the SHG susceptibilities in- 
volving EQ and/or MD processes may lead to signals of appreciable magnitude, especially in the vicinity of electronic resonances. Intuitively, one may expect that EQ processes are more important in centrosymmetric diamagnetic materials, see, e.g., Ref. 13 and references therein. MD processes are expected to be more important in paramagnetic or magnetically ordered materials. However, this general rule does not exclude other possibilities. For example, nonlinear optical studies of diamagnetic $\mathrm{C}_{60}$ fullerene films revealed $\mathrm{MD}$, ED, and EQ mechanisms. ${ }^{14,15}$ Other interesting examples are the observations of SHG due to EQ in artificial photonic structures, ${ }^{16}$ and due to MD resonances in centrosymmetric antiferromagnets ${ }^{3,9}$ and in metamaterials. ${ }^{17}$

An important activity of research on SHG and higher order harmonics generation is the search for new mechanisms of nonlinear interaction of light in centrosymmetric and noncentrosymmetric materials, induced by crystallographic and magnetic phase transitions or external perturbations that break the spatial inversion or time reversal symmetry. Breaking of the spatial inversion symmetry at the paraelectricferroelectric phase transition in $\mathrm{BaTiO}_{3}$ makes the ED contribution to SHG allowed. ${ }^{18}$ An ED SHG mechanism may arise at magnetic phase transitions due to particular types of spin ordering, as is the case for crystallographically symmetric $\mathrm{Cr}_{2} \mathrm{O}_{3} \cdot{ }^{19}$

At the very beginning of studies of induced SHG, optical SHG was observed in centrosymmetric calcite $\mathrm{CaCO}_{3}$ subject to a DC electric field, ${ }^{20}$ which is a polar vector. A DC electric field is the most straightforward and efficient perturbation for inducing a bulk ED contribution to SHG. Another perturbation for inducing ED-SHG is inhomogeneous strain, for example due to the lattice mismatch between a substrate and a thin film grown on it. ${ }^{21-24}$

A DC magnetic field applied to a medium may also induce new contributions to SHG. However, this mechanism is radically different from the action of an electric field or inhomogeneous strain, because the transformation properties of a magnetic field are those of an axial vector. Magneticfield-induced SHG process is expected to be much weaker than ED driven SHG. Nevertheless, relatively strong magnetic effects due to spontaneous magnetization and/or magnetic ordering are observed in different ferromagnetic and antiferromagnetic materials. Magnetization-induced SHG was found in reflection from ferromagnetic iron surface ${ }^{25}$ and also in other materials as discussed in several reviews. ${ }^{3,7,8}$ In most cases the observed SHG signals could be related to the influence of the magnetization on the intrinsic ED-SHG, due to inversion symmetry breaking either in the bulk or at the surface.

A large class of semiconductors crystallizing in the zinc-blende (sphalerite), wurtzite, chalcopyrite, and other noncentrosymmetric structures allow for SHG in the ED approximation. However, nonlinear optical studies of semiconductors using SHG have been, for quite a long time, limited to specific singular wavelengths of commercially available lasers. Examples of SHG spectroscopic studies in semiconductors are still scarce and limited to narrow spectral ranges. ${ }^{26-28}$ Cubic semiconductors such as GaAs and CdTe, though possessing high-SHG susceptibilities, do not allow one to fulfill phase matching conditions so that their imple- mentation in application devices is not efficient. On the other hand, striking progress has been achieved in the growth of noncubic semiconductor chalcopyrite single crystals such as $\mathrm{ZnGeP}_{2}, \mathrm{AgGaS}_{2}$, and $\mathrm{CdGeAs}_{2}$. For them phase matching can be achieved, which enables their use in continuously tunable laser sources over wide infrared spectral ranges. ${ }^{29-32}$

Detailed spectroscopic SHG studies of semiconductors at low temperatures, with high spectral resolution, and in applied magnetic field were initiated only in recent years. It was discovered that the application of a magnetic field to the noncentrosymmetric diamagnetic semiconductors GaAs and CdTe, for which SHG is allowed in ED approximation, induces new optical nonlinearities. ${ }^{33,34}$ These were attributed to Landau-level orbital quantization of the band energy spectrum. In the diluted magnetic semiconductor $(\mathrm{Cd}, \mathrm{Mn}) \mathrm{Te}$ the SHG response was found to be induced by the giant spinsplitting of electronic states. ${ }^{35,36}$

Very recently a type of spin-induced SHG in the centrosymmetric magnetic semiconductors EuTe and EuSe was observed. ${ }^{37}$ It was demonstrated to be related to the nonlinear optical susceptibility $\chi^{e e m}$ caused by a MD contribution from spontaneous ferromagnetic or induced paramagnetic magnetization. This observation opens new opportunities for studying the electronic and magnetic structures of centrosymmetric semiconductors. Further experimental and theoretical efforts are required for a deeper understanding of the mechanisms involved in the SHG processes in centrosymmetric materials. In this paper, we present new experimental results on SHG in EuTe and EuSe and analyze them in the frame of a recently developed microscopic theory. ${ }^{38}$

The paper is organized as follows. In Sec. II, we describe the crystallographic and electronic structures as well as the magnetic, optical and magneto-optical properties of $\mathrm{EuX}$ compounds. Section III addresses a symmetry description of SHG. Experimental details are given in Sec. IV, which is followed by Sec. V where the experimental results are presented. Section VI gives the microscopic theoretical modeling of the SHG and comparison of the experimental data with the microscopic calculations. Section VII concludes the paper.

\section{PROPERTIES OF EUROPIUM CHALCOGENIDES}

The europium chalcogenides $\mathrm{Eu} X$ are wide band gap magnetic semiconductors, which were discovered in the early 1960s. They are characterized by several unique electronic and magnetic properties, because of which they attract continuous interest for academic research as well as for device applications. $\mathrm{Eu} X$ have been suggested for applications in spin-filter devices based on $\mathrm{EuO}^{39-43} \mathrm{EuS}^{44-50}$ or $\mathrm{EuSe}^{51}$ tunnel junctions. These tunnel junctions provide spinpolarized electrons due to different barrier heights for electrons with opposite spin orientations. ${ }^{52,53}$ Due to the giant Faraday rotation EuS and EuSe films can be applied also for high-resolution magneto-optical imaging of e.g., the flux distribution in superconductors. ${ }^{54}$ Recently it was shown, that $\mathrm{EuO}$ can be epitaxially grown on $\mathrm{Si}^{55}$ and $\mathrm{GaN},{ }^{56}$ which opens new possibilities for device realization. 


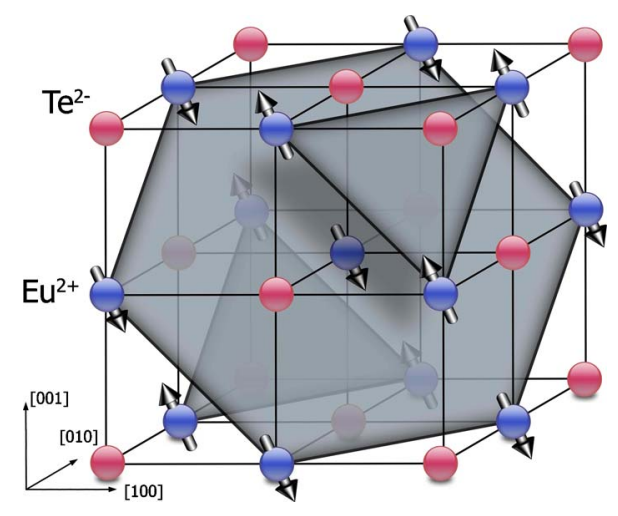

FIG. 1. (Color online) Blue (with arrows) and red (without arrows) spheres are the $\mathrm{Eu}^{2+}$ and $\mathrm{Te}^{2-}$ ions, respectively. Spins aligned in parallel are marked by gray planes. Spins of neighboring planes are aligned antiferromagnetically below the Néel temperature.

\section{A. Crystallographic structure}

The europium chalcogenides $\operatorname{Eu} X(X=\mathrm{O}, \mathrm{S}, \mathrm{Se}$, and $\mathrm{Te})$ crystallize in the centrosymmetric cubic rock salt structure with $m 3 m\left(O_{h}\right)$ symmetry. Figure 1 shows the crystal structure of EuTe with a lattice constant $a_{0}=6.598 \AA$ (see Table I). The face centered cubic (fcc) lattice contains a two-atom basis given by $\mathrm{Eu}^{2+}$ ions at $(1 / 2,1 / 2,1 / 2)$ and $\mathrm{Te}^{2-}$ ions at $(0,0,0)$. The divalent $\mathrm{Eu}^{2+}$ ions are octahedrally surrounded by six $\mathrm{Te}^{2-}$ ions forming a strong ionic binding, which results in empty $5 d$ states of europium and completely filled $p$ orbitals of tellurium.

\section{B. Electronic band structure}

The electronic level structure of the $\mathrm{Eu} X$ is of principal importance for understanding their magnetic, optical and magneto-optical properties. Due to the complexity of the Eu atom with many core electrons, first-principle self-consistent calculations of the electronic structure in $\mathrm{Eu} X$ with good enough accuracy are not yet possible, and interpretations of the experimental data have relied on a semiempirical BornHaber cycle (see Ref. 57). Because the Born-Haber cycle gives only an approximate and rough picture, it is necessary to refine our understanding of the energy band structure on the basis of direct experimental evidence.

The electronic properties of the europium chalcogenides are determined by the $4 f^{7}$ electrons of the $\mathrm{Eu}^{2+}$ ions with spin $S=7 / 2 .^{58,63,64}$ It is generally accepted that the $\mathrm{Eu}^{2+}$ $4 f^{7}\left({ }^{8} S_{7 / 2}\right)$ atomic states constitute the valence level in $\mathrm{Eu} X$,

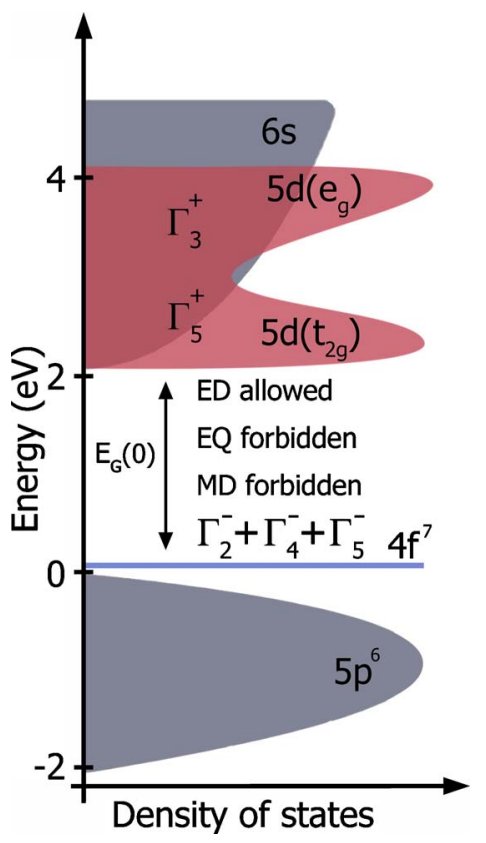

FIG. 2. (Color online) Schematic picture of the energy band structure of EuTe. For single photon processes in the vicinity of the band gap only ED transitions are allowed.

which lies within the energy gap, directly on top of a $2-3 \mathrm{eV}$ wide valence band. This valence band below the $4 f^{7}\left({ }^{8} S_{7 / 2}\right)$ level is formed by $n p$ orbitals of the chalcogen, where $n=2$, 3, 4, and 5 for $\mathrm{O}, \mathrm{S}, \mathrm{Se}$, and Te, respectively, based on $\mathrm{EuX}$ photoemission studies, ${ }^{65}$ and consistent with magneto-optical data. ${ }^{66-73}$ The lowest-energy conduction band has a narrow width of $\sim 100 \mathrm{meV},{ }^{61,62}$ so that it can be described by a tight-binding model. The basis set of the tight-binding wave functions consists of the $5 d$ orbitals of the Eu atoms, split by the octahedral crystal field into a twofold degenerate lowenergy $5 d\left(t_{2 g}\right)$ state, and a $5 d\left(e_{g}\right)$ state at about $2 \mathrm{eV}$ higher energy. This assertion is consistent with magneto-optical data. ${ }^{66-73}$ It is also generally assumed that a broad conduction band with $6 s$ character is nearly resonant with the $5 d\left(t_{2 g}\right)$ states, however, electric-dipole coupling of the $4 f^{7}\left({ }^{8} S_{7 / 2}\right)$ state to this $6 s$ band is forbidden, which is therefore optically dark. The single-particle electronic structure in EuTe is shown schematically in Fig. 2.

The electrons in the europium $4 f^{7}$ level are strongly localized around the Eu atom to which they belong, because of the Coulomb attraction by the unscreened Eu core, and they are shielded from the surrounding by electrons occupying the $5 s$ and $5 p \mathrm{Eu}$ orbitals. Therefore, the $4 f^{7}$ electrons are in

TABLE I. Parameters of EuSe and EuTe (Refs. 38 and 58-62)

\begin{tabular}{|c|c|c|c|c|c|c|c|c|c|}
\hline $\operatorname{Eu} X$ & $\begin{array}{c}a_{0} \\
(\AA)\end{array}$ & Order & $\theta(K)$ & $T_{N, C}(K)$ & $J_{1}(K)$ & $J_{2}(K)$ & $\begin{array}{c}E_{g} \\
(\mathrm{eV})\end{array}$ & $\begin{array}{l}J_{d f} S \\
(\mathrm{eV})\end{array}$ & $\begin{array}{c}\lambda_{4 f} \\
(\mathrm{meV})\end{array}$ \\
\hline \multirow[t]{3}{*}{ EuSe } & 6.195 & AFM & 9 & 4.60 & 0.13 & -0.11 & 2.092 & 0.104 & 14.0 \\
\hline & & FIM & & 3.6 & & & & & \\
\hline & & AFM & & 2.8 & & & & & \\
\hline EuTe & 6.598 & AFM & -6 & 9.58 & 0.03 & -0.15 & 2.321 & 0.130 & 9.6 \\
\hline
\end{tabular}


effect unaffected by the crystal environment, and can be described by the wave functions of an isolated Eu atom. According to Hund's rules, at $T=0 \mathrm{~K}$ all seven electrons in the localized $4 f$ shell of the $\mathrm{Eu}^{2+}$ ion will have parallel spins, thus their total electronic spin quantum number is $S=7 / 2$, which by the Pauli exclusion principle leads to a state with total orbital momentum quantum number $L=0$. The standard spectroscopic notation of this state is, therefore, $4 f^{7}\left({ }^{8} S_{7 / 2}\right)$. The excitation of lowest energy corresponds to the transfer of one electron from the $4 f^{7}\left({ }^{8} S_{7 / 2}\right)$ state into the $5 d\left(t_{2 g}\right)$ conduction band. We shall denote by $X$ the single-particle electronic states belonging to the $5 d\left(t_{2 g}\right)$ conduction band. In the tight-binding model, the electronic wave function of an $X$ state is given by

$$
X(\boldsymbol{k}, \boldsymbol{r})=\frac{1}{\sqrt{\mathcal{N}}} \sum_{\boldsymbol{R}} e^{i \boldsymbol{R} \cdot \boldsymbol{k}} \phi(\boldsymbol{r}-\boldsymbol{R}) .
$$

Here, $\phi(\boldsymbol{r})$ represents one of the $5 d\left(t_{2 g}\right)$ europium orbitals $\left(d_{x y}, d_{y z}\right.$ or $\left.d_{z x}\right), \boldsymbol{R}$ is the position vector of an Eu atom in the lattice, and $\mathcal{N}$ is the number of lattice sites inside the BornKarmán volume.

Once an electron is transferred from the $4 f^{7}\left({ }^{8} S_{7 / 2}\right)$ ground state into an $X$ exited state, six electrons remain in the $4 f$ level. Due to the residual Coulomb interaction among them, this six-electron ensemble forms a minimum-energy state with total spin quantum number $S=3$ and total orbital quantum number $L=3$. The spin-orbit interaction couples the orbital $L$ and spin $S$ angular momenta to a state with total angular momentum $J$, where $J$ can take values $J$ $=0,1, \ldots, 6$. The energy of these 6 core electrons depends on $J$ according to the Landé interval rule:

$$
E_{J}=\frac{1}{2} \lambda_{4 f} J(J+1),
$$

where $\lambda_{4 f}$ is the spin-orbit coupling constant.

The standard spectroscopic notation for $L=3, S=3$, and total momentum $J$ is ${ }^{7} F_{J}$. Including the magnetic quantum number $M=-J, \ldots, 0, \ldots, J$ for a fixed $J$, the lowest energy excited state, in which a hole is created at an Eu lattice site and an electron is created in an $X$ conductive state, can be represented by the notation ${ }^{7} F_{J M} X$, used henceforward.

\section{Magnetic properties}

The magnetic properties of the europium chalcogenides are determined by the ground state of the $\mathrm{Eu}^{2+}$ ions in which the $4 f^{7}$ electrons with spin $S=7 / 2$ are involved. ${ }^{58,63,64} \mathrm{Eu} X$ are classical Heisenberg magnets where the competition between the nearest neighbor (NN) exchange integral $J_{1}$ and the next-nearest neighbor (NNN) integral $J_{2}$ results in magnetic phase diagrams that may include antiferromagnetic (AFM), ferrimagnetic (FIM), and ferromagnetic (FM) ordering, as well as a paramagnetic phase at elevated temperatures. ${ }^{58,59}$ Since the $4 f$ electrons are spatially localized at the Eu-core, no overlap of $4 f$-wave functions of neighboring Eu-ions is expected. Therefore, the exchange interaction must be indirect. The ferromagnetic NN interaction $J_{1}$ depends strongly on the interatomic distance, as one can see from Table I. The antiferromagnetic NNN interaction is denoted by $J_{2}$. The values of exchange integrals, Néel temperature $\left(T_{N}\right)$ and Curie temperature $(\theta)$ are listed in Table I. The unique magnetic properties and complicated magnetic phase diagrams of $\mathrm{Eu} X$ are caused by the varying ratios of the exchange parameters $J_{1}$ and $J_{2}$ and by the competition of the exchange interactions between magnetic ions with their interaction with an external magnetic field.

In EuTe $\left|J_{2}\right|>\left|J_{1}\right|$ and therefore this compound shows antiferromagnetism below $T_{N}=9.58 \mathrm{~K} .{ }^{58}$ Absorption and Faraday rotation measurements reveal a critical field of $B_{\text {sat }}=7.2 \mathrm{~T}$ above which it becomes ferromagnetically saturated. ${ }^{60}$ The $\mathrm{Eu}^{2+}$ spins are parallel within the (111) planes and adjacent planes have alternating spin orientation $[\uparrow \downarrow \uparrow \downarrow]$, see Fig. 1. Below $T_{N}$ the magnetic ordering in EuTe can be characterized by the magnetic moments $\mathbf{m}_{1}$ and $\mathbf{m}_{2}$ of the two sublattices with $\left|m_{1}\right|=\left|m_{2}\right|$. To describe the magnetic behavior of an antiferromagnet in external magnetic field we introduce a $\mathrm{FM}$ vector $\mathbf{F}=\mathbf{m}_{1}+\mathbf{m}_{2}$ and an $\mathrm{AFM}$ vector $\mathbf{A}$ $=\mathbf{m}_{1}-\mathbf{m}_{2}$. Though $\mathbf{F}$ and $\mathbf{A}$ are composed of the same vectors $\mathbf{m}_{1}$ and $\mathbf{m}_{2}$, their transformation properties under space inversion and time reversal operations are different. These distinctions are important for the analysis of their contributions to SHG process, as discussed below in Sec. III.

The magnetic behavior of EuSe is more complicated and is called metamagnetic. ${ }^{58,59}$ In this case the ferromagnetic and antiferromagnetic exchange integrals $J_{1}$ and $J_{2}$ have comparable magnitudes so that the material displays several magnetic phases, which are strongly sensitive to temperature and external magnetic field strength. At zero magnetic field EuSe is antiferromagnetic below $T_{N}=4.6 \mathrm{~K}$ with adjacent spin planes aligned as $[\uparrow \uparrow \downarrow \downarrow]$. A ferrimagnetic phase is found below $T_{C}=3.6 \mathrm{~K}$ with $[\uparrow \uparrow \downarrow]$ spin plane structure. For temperatures lower than $T_{N}=2.8 \mathrm{~K}$ the ordering changes to another antiferromagnetic phase with $[\uparrow \downarrow \uparrow \downarrow]$ plane alignment. Even a weak external magnetic field is sufficient to switch between the phases: the two antiferromagnetic phases turn into a ferrimagnetic phase and the ferrimagnetic phase into a ferromagnetic phase with increasing field strength. In a magnetic field above a critical value of $0.2 \mathrm{~T}$ EuSe is in a FM phase $(2.2 \mathrm{~K}<T<5.8 \mathrm{~K}){ }^{59}$

\section{Optical and magneto-optical properties}

The optical and magneto-optical properties of the europium chalcogenides are very distinctive, and some of these properties are unmatched by any other magnetic semiconductor. As discussed the optical band gap is formed by the lowest energy excitation corresponding to the transfer of an electron from the $4 f^{7}\left({ }^{8} S_{7 / 2}\right)$ state into the $5 d\left(t_{2 g}\right)$ conduction band. When subjected to an external magnetic field, EuTe and EuSe exhibit a gigantic red shift (as high as $15 \mathrm{meV} / \mathrm{T}$ in EuTe) of the optical absorption threshold. ${ }^{58,63}$ The red shift of the optical band gap in EuSe is characterized by a huge gyromagnetic factor up to $18000 .^{74}$ The photoluminescence of EuTe undergoes the largest energy redshift ever observed in semiconductors. ${ }^{75}$ The Faraday effect is also extraordinary in $\mathrm{Eu} X$ with Verdet constants as high as $10^{6} \mathrm{deg} / \mathrm{cm} .^{76-81}$

The magneto-optical data, from which the mentioned features of the electronic structure were extracted, were re- 
corded on bulk EuX crystals, and provide experimental support for the $4 f^{7}\left({ }^{8} S_{7 / 2}\right) \rightarrow 5 d\left(t_{2 g}\right)$ model. However, looking in more detail, the $4 f^{7}\left({ }^{8} S_{7 / 2}\right) \rightarrow 5 d\left(t_{2 g}\right)$ model has failed to explain several experimental observations: the model predicts narrow spectral lines for the band-edge optical absorption, ${ }^{72}$ which were not observed experimentally. This disagreement was attributed to many-body effects, not included in the single-particle $4 f^{7}\left({ }^{8} S_{7 / 2}\right) \rightarrow 5 d\left(t_{2 g}\right)$ model (see discussions in Ref. 57, Sec. 3.2.2, Ref. 72, Sec. D, and Ref. 73, Sec. I.5B).

More recently, molecular-beam epitaxy (MBE) was used to fabricate thick $(1-4 \mu \mathrm{m})$ epitaxial layers of stoichiometric $\mathrm{Eu} X$, which show high purity and structural quality. In such EuTe and EuSe layers at helium temperatures, the absorption spectra showed narrow dichroic features in magnetic field, in good agreement with theoretical predictions based on the single-particle $4 f^{7}\left({ }^{8} S_{7 / 2}\right) \rightarrow 5 d\left(t_{2 g}\right)$ model. ${ }^{61,62}$ The disappearance of narrow absorption lines when the magnetic field is turned off can be explained in the framework of the $4 f^{7}\left({ }^{8} S_{7 / 2}\right) \rightarrow 5 d\left(t_{2 g}\right)$ model, when the breakup of the sample into an ensemble of magnetic domains is taken into account. ${ }^{62,82,83}$ In the present work, we shall use the $4 f^{7}\left({ }^{8} S_{7 / 2}\right) \rightarrow 5 d\left(t_{2 g}\right)$ model to interpret the optical nonlinearities observed in $\mathrm{EuX}$.

An electron in a $5 d\left(t_{2 g}\right)$ Bloch state is subject to a strong exchange interaction with the $4 f^{7}\left({ }^{8} S_{7 / 2}\right)$ electrons at the $\mathrm{Eu}^{2+}$ lattice sites (the so-called $d-f$ exchange interaction), which can be described by the Hamiltonian ${ }^{60}$

$$
\mathcal{H}_{d f}=-J_{d f} \sum_{n \mu \nu} a_{n \mu}^{\dagger} a_{n \nu} \boldsymbol{S}_{n} \cdot \boldsymbol{\sigma}_{\mu \nu}
$$

where $J_{d f}$ is the $d-f$ exchange interaction constant, $a_{n \mu}^{\dagger}$ is the creation operator of a Wannier function with spin $\mu$, centered at the $n$-th Eu atom, $\boldsymbol{\sigma}$ is a Pauli spin operator, and $S_{n}$ is the spin of the $n$-th Eu site. In zero magnetic field, when the $\mathrm{Eu}^{2+}$ spins are ordered antiferromagnetically, $\mathcal{H}_{d f}$ averages to zero, but if a strong external magnetic field imposes ferromagnetic order, $\mathcal{H}_{d f}$ leads to a maximum value of $J_{d f} S$, where $S=7 / 2$. For intermediate magnetic fields, the distribution of the $\mathrm{Eu}^{2+}$ spins can be calculated using molecular field theory. ${ }^{82}$ The $d-f$ exchange interaction is precisely the source of the band-gap shrinkage in EuTe and EuSe. For a thin EuTe layer subjected to an in-plane magnetic field $B$, the field dependence of the optical bandgap in EuTe can be described by ${ }^{60}$

$$
E_{g}(B)=E_{g}(0)-J_{d f} S \times\left\{\begin{array}{cl}
\left(\frac{B}{B_{\text {sat }}}\right)^{2} & \text { if } B \leq B_{\text {sat }} \\
1, & \text { if } B>B_{\text {sat }}
\end{array},\right.
$$

where $B_{\text {sat }}=7.2$ T..$^{60,84}$

In addition to the redshift, the application of a magnetic field also gives rise to the Zeeman effect, which lifts the $2 M+1$-fold degeneracy of the ${ }^{7} F_{J M}$ core, and the up/down spin degeneracy of the $5 d\left(t_{2 g}\right)$ Bloch state. However, the electron's Landé gyromagnetic factor is $g_{J}=2$ for both the $4 f^{7}\left({ }^{8} S_{7 / 2}\right)$ and the $5 d\left(t_{2 g}\right)$ states, and $g_{J}=3 / 2$ for the ${ }^{7} F_{J M}$ states, hence the Zeeman interaction leads to a level shift of

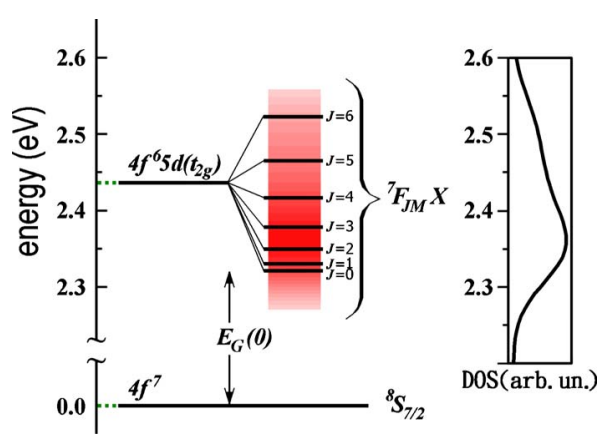

FIG. 3. (Color online) Energy spectrum of the band-edge electronic excitations in EuTe and EuSe. The scale applies to EuTe at $B=0$. The color tone of the shaded area reflects the magnitude of the density of states, shown in the right panel.

only a few tens of $\mu \mathrm{eV} / \mathrm{T}$, which is negligible in comparison with the $d-f$ redshift of $15 \mathrm{meV} / \mathrm{T}$, and we will therefore disregard it.

The energy of the lowest energy electronic excitation depends only on the quantum numbers $J$ and $X$, and is given by

$$
E_{J X}=E_{g}(B)+E_{J}+\epsilon_{X}(\boldsymbol{k}), \quad J=0, \ldots, 6 .
$$

Here $\epsilon_{X}(\boldsymbol{k})$ is the energy dispersion of the $5 d\left(t_{2 g}\right)$ narrow conduction band, $E_{g}(B)$ is the magnetic field dependent band gap of $\mathrm{Eu} X$, given by Eq. (5) with $\left(E_{g}(0)=2.321 \mathrm{eV}\right.$ for EuTe $^{61,62}$ and $E_{g}(0)=2.092 \mathrm{eV}$ for EuSe. $\left.{ }^{61}\right) \lambda_{4 f}$ is the Landé spin-orbit constant: $\lambda_{4 f}=9.6 \mathrm{meV}$ for EuTe and $\lambda_{4 f}$ $=14 \mathrm{meV}$ for EuSe ${ }^{61}$

The lowest-energy optically active electronic excitations of $\mathrm{Eu} X$ are shown for EuTe in Fig. 3. The $4 f^{7}\left({ }^{8} S_{7 / 2}\right)$ excitation scheme in Fig. 3 was used successfully to model the dichroic optical absorption threshold in EuTe and EuSe, using the parameters given in Table I, yielding a width of each individual $5 d\left(t_{2 g}\right)$ conduction band of about $100 \mathrm{meV} .^{61,62}$ Therefore the energy of electronic excitations into a given $5 d\left(t_{2 g}\right)$ conduction band, which leave the $\mathrm{Eu}^{3+}$ atom in a given $J$ state, overlaps in energy with excitations into the same conduction band, but for a different value of $J$, and thus a continuum of excitations is formed. As a result, the width of the continuum is nearly $300 \mathrm{meV}$, as seen in Fig. 3 .

\section{SYMMETRY CONSIDERATION OF SECOND HARMONIC GENERATION}

\section{A. Polarization selection rules for SHG}

Coherent three-photon SHG processes become strongly anisotropic even in cubic crystals which are isotropic in linear optics. A particularly characteristic property of the material under study is the rotational anisotropy of the SHG signal as function of the polarizations of the incident and outgoing light beams. Based on the crystallographic symmetry and the band structure parity, we discuss here the selection rules for different SHG processes. The centrosymmetric crystal structure of $\mathrm{Eu} X$ leads to an important restriction for the SHG process, because the strongest ED-SHG contribution related to the $\chi^{e e e}$ nonlinear susceptibility is forbidden. Further information can be revealed by a more detailed 
analysis of the parity of the involved bands and the possible optical excitation processes. The odd symmetry of the $4 f$ valence band is described by the $\Gamma_{2}^{-}+\Gamma_{4}^{-}+\Gamma_{5}^{-}$representation of the cubic $\mathrm{O}_{h}$ group. ${ }^{85}$ The symmetry of the even conduction band $5 d\left(t_{2 g}\right)$ is $\Gamma_{5}^{+}$and for the $5 d\left(e_{g}\right)$ band it is $\Gamma_{3}^{+}$. Therefore in linear optics the electric-dipole-induced transitions characterized by $\Gamma_{4}^{-}$symmetry are allowed due to their odd parity.

Since in the SHG process two incident photons and one outgoing photon are involved, the strongest ED excitation requires two $\Gamma_{4}^{-}$transitions. It has therefore again even parity and in the present case is parity forbidden. The higher order SHG processes given by the electric quadrupole $\Gamma_{3}^{+}+\Gamma_{5}^{+}$and the magnetic dipole $\Gamma_{4}^{+}$contributions, have even parity and therefore cannot induce a resonant transition in linear optics as well as in nonlinear optics. For resonant excitation an odd SHG process is needed. This can only be provided by the MD process as ${ }^{1,2}$

$$
P_{i}^{C M D}(2 \omega)=i \varepsilon_{0} \chi_{i j k}^{e e m}(-2 \omega ; \omega, \omega) E_{j}(\omega) B_{k}(\omega),
$$

consisting of an even magnetic dipole transition and an odd electric dipole excitation. ${ }^{86}$ Here, $E_{j}(\omega)$ and $B_{k}(\omega)$ are the electric and magnetic fields of the fundamental light wave, respectively. In the following this polarization is called crystallographic magnetic dipole (CMD) contribution. $\chi_{i j k}^{e e m}(-2 \omega ; \omega, \omega)$ is an axial third-rank tensor allowed in any medium. ${ }^{87}$

Note that the same type of tensor $\chi_{i j k}^{e e m}(-\omega ; \omega, 0)$ describes the magneto-optical Faraday effect, if in Eq. (7) the field $B_{k}(\omega)$ is replaced by an external magnetic field $B_{k}(0)$, and the polarization $P_{i}(\omega)$ is excited at the fundamental frequency. In view of the giant Faraday effect in $\mathrm{EuX}$ (Ref. 77) a significant MD contribution to the SHG is expected. ${ }^{86}$ In bulk EuX compounds with point group $m 3 m$ the tensor $\chi_{i j k}^{e e m}$ has only one nonvanishing independent component $x y z(c 3)$ $=-x z y(c 3),{ }^{87}$ which, however, for all crystal orientations does not lead to any SHG intensity $I(2 \omega) \propto\left|\mathbf{P}^{C M D}(2 \omega)\right|^{2}$, because of the alternating sign for every axis permutation. The notation of the type $x y z(c 3)$ denotes the three distinct cyclic permutations of $x y z$.

For layers of EuTe and EuSe grown on a (111) $\mathrm{BaF}_{2}$ substrate (see Sec. IV) there is a small mismatch between the sample and substrate lattice constants resulting in a weak trigonal distortion along the [111] axis. Evidently this causes a symmetry reduction of thin EuTe and EuSe layers to the trigonal centrosymmetric point group $\overline{3} \mathrm{~m}$ in the proximity of the interface. Nevertheless the centrosymmetry remains and ED as well as EQ contributions cannot be induced. The tensor $\chi_{i j k}^{e e m}$ in this point group has one independent component $x y z(6),{ }^{87}$ which may produce a small crystallographic MD contribution to the SHG signal, if the wave vector of the incident light beam $\mathbf{k}$ is not parallel to one of the fundamental crystal axes $\mathbf{x}, \mathbf{y}$, or $\mathbf{z}$.

A new type of nonlinear polarization can be induced if the crystal symmetry is broken by either magnetic field or magnetic ordering, both of which are described here by the magnetic parameter $\mathbf{M}(0)$. The nonlinear polarization by the induced magnetic dipole (IMD) is

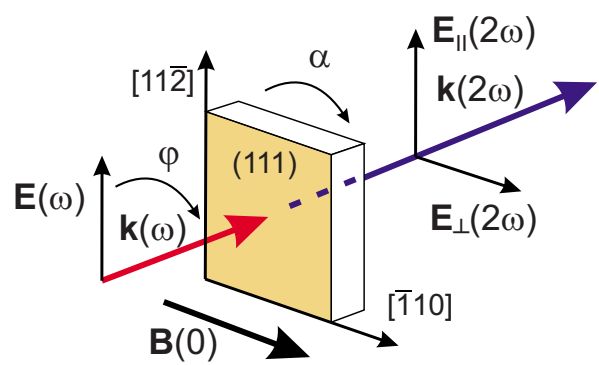

FIG. 4. (Color online) Sketch of the experimental geometry in the SHG studies.

$$
P_{i}^{I M D}(2 \omega)=\varepsilon_{0} \chi_{i j k l}^{e e m m}(-2 \omega ; \omega, \omega, 0) E_{j}(\omega) B_{k}(\omega) M_{l}(0),
$$

where $\chi_{i j k l}^{e e m m}$ is a polar fourth-rank tensor ${ }^{87}$ with nonvanishing tensor components

$$
\begin{gathered}
\chi_{x x y y}(x: 3)=\chi_{y y x x}(y: 3)=\chi_{x x z z}(x: 3)=\chi_{y y z z}(y: 3) \\
=\chi_{z z y y}(y: 3)=\chi_{z z x x}(y: 3), \\
\chi_{x x x x}=\chi_{y y y y}=\chi_{z z z z} .
\end{gathered}
$$

Here, the notations of type $x x y y(x: 3)$ denote the three distinct components which may be obtained from the component $x x y y$ by keeping the first index fixed and permutating all the others.

\section{B. SHG rotational anisotropy}

The polarization dependence of the SHG signal, namely its azimuthal rotational anisotropy, gives in-depth information on the symmetries and involved ED, MD, and/or EQ mechanisms of nonlinear light-matter interaction. For calculating the rotational anisotropy we used the following procedure. The rotational SHG anisotropy is detected for simultaneous rotation of linear polarizers for the fundamental and SHG light. For calculating it, the components given in Eqs. (7) and (8) with respect to the crystal coordinate system have to be transformed into the laboratory coordinate system, accounting for the experimental geometry. This geometry is shown in Fig. 4. The laboratory coordinate system is spanned by the light wave vectors $\mathbf{k}(\omega) \| \mathbf{k}(2 \omega)$, the electric field $\mathbf{E}(\omega)$ and the external magnetic field $\mathbf{B}(0)$. The crystallographic coordinate system is defined by the $x, y$, and $z$ axes. Thereby $\varphi$ denotes the angle between the direction of the magnetic field $\mathbf{B}(\omega)$ of the fundamental light and the external magnetic field, and $\alpha$ is the sample azimuthal angle. For $\alpha=0$ and $\varphi=0$ this leads to $\mathbf{E}(\omega) \|[11 \overline{2}]$ and $\mathbf{B}(\omega)\|[\overline{1} 10]\| \mathbf{B}(0)$. Then the transformed tensors have to be inserted in the equations for the polarizations. Since $\mathrm{Eu} X$ samples are grown on (111) substrates and the experiments were performed at normal light incidence with $\mathbf{k} \|[111]$, only this geometry is considered. For the crystallographic contribution to the SHG intensity $I(2 \omega) \propto\left|\mathbf{P}^{C M D}(2 \omega)\right|^{2}$ in the parallel configuration $\mathbf{E}(2 \omega) \| \mathbf{E}(\omega)$ one obtains

$$
I_{\|}(2 \omega) \propto\left|\chi_{x y z} \cos 3(\varphi-\alpha)\right|^{2},
$$

and for the crossed arrangement $\mathbf{E}(2 \omega) \perp \mathbf{E}(\omega)$, 

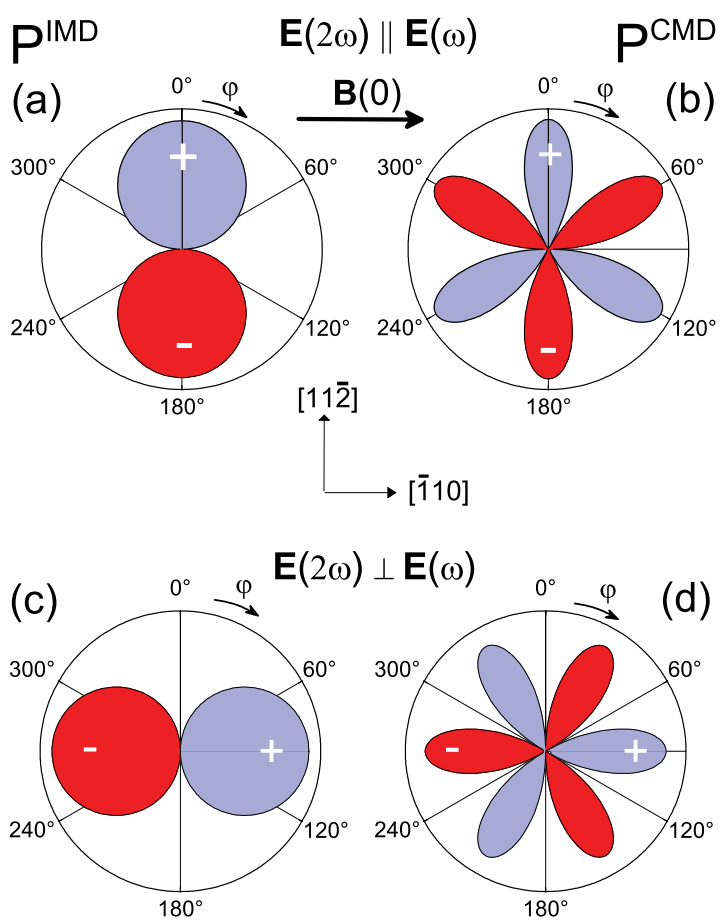

FIG. 5. (Color online) Polar plots of SHG intensity calculated for the IMD and CMD nonlinear polarizations in case of a $\overline{3} m$ symmetry.

$$
I_{\perp}(2 \omega) \propto\left|\chi_{x y z} \sin 3(\varphi-\alpha)\right|^{2} .
$$

As shown in Figs. 5(b) and 5(d) one obtains sixfold rotation patterns as expected for the light propagating along the threefold symmetry axis. The $I(2 \omega) \propto\left|\mathbf{P}^{I M D}(2 \omega)\right|^{2}$ contribution induced by an external magnetic field can be modeled by

$$
I_{\|}(2 \omega) \propto\left|\frac{M}{6}\left(\chi_{x x x x}+5 \chi_{x x y y}-\chi_{x y x y}-\chi_{x y y x}\right) \cos \varphi\right|^{2},
$$

in the case of $\mathbf{E}(2 \omega) \| \mathbf{E}(\omega), \alpha=0$. For $\mathbf{E}(2 \omega) \perp \mathbf{E}(\omega), \alpha=0$ one obtains

$$
I_{\perp}(2 \omega) \propto\left|\frac{M}{6}\left(\chi_{x x x x}-\chi_{x x y y}+5 \chi_{x y x y}-\chi_{x y y x}\right) \sin \varphi\right|^{2},
$$

where $M$ is the magnetization. Most important is that this magnetic contribution shows twofold patterns in Figs. 5(a) and $5(\mathrm{c})$ and does not depend on the orientation of the sample, but only on the direction of the magnetization.

As noted above with respect to an ideal bulk crystal (or a strain-free film), for $\mathbf{k}$ parallel to one of the main crystal axis $\mathbf{x}, \mathbf{y}$, or $\mathbf{z}$ the crystallographic MD contribution vanishes. But for the chosen experimental geometry and samples in an external magnetic field the crystallographic contribution does not vanish, and therefore the interference between the IMD [Eq. (8)] and CMD [Eq. (7)] contributions should be accounted for

$$
I(2 \omega) \propto\left|\mathbf{P}^{I M D}\right|^{2}+\left|\mathbf{P}^{C M D}\right|^{2} \pm 2\left|\mathbf{P}^{I M D} \mathbf{P}^{C M D}\right| .
$$

Here, the signs \pm correspond to opposite orientations of $\mathbf{M}(0)$. The SHG intensities in parallel and perpendicular configurations of the fundamental and SHG light polarizations for $\alpha=0$ are

$$
\begin{aligned}
I_{\|}(2 \omega) \propto & \pm \frac{M}{6}\left(\chi_{x x x x}+5 \chi_{x x y y}-\chi_{x y x y}-\chi_{x y y x}\right) \cos \varphi \\
& -\left.\chi_{x y z} \cos 3 \varphi\right|^{2}, \\
I_{\perp}(2 \omega) \propto & \mid \pm \frac{M}{6}\left(\chi_{x x x x}-\chi_{x x y y}+5 \chi_{x y x y}-\chi_{x y y x}\right) \sin \varphi \\
& +\left.\chi_{x y z} \sin 3 \varphi\right|^{2} .
\end{aligned}
$$

The phases of the rotation pattern in Fig. 5 are denoted by \pm to distinguish between possible constructive and destructive interference geometries.

\section{EXPERIMENTAL TECHNIQUE AND SAMPLES}

To generate the optical second harmonic, we used a pulsed Nd:YAG laser with a wavelength of $355 \mathrm{~nm}$ and a pulse duration of $8 \mathrm{~ns}$, emitted at a repetition rate of $10 \mathrm{~Hz}$, to pump a beta barium borate (BBO)-based optical parametric oscillator (OPO). The tunable type-II OPO allowed one to access a wide spectral range (410-2500 nm) by nonlinear spectroscopy with a typical pulse power of $20 \mathrm{~mJ}$ and a narrow linewidth of about $0.4 \mathrm{meV}$. A Glan-Thomson prism and quarter and or half-wave plates were used to set the required light polarization at the fundamental frequency. The laser light beam of the OPO was sent through the sample, and the signal generated at the second harmonic frequency was studied. Optical filters were used to separate the fundamental wave and the SHG signal spectrally.

An analyzer enables polarization dependent detection of the nonlinear signal. The light is focused onto a spectrometer, which is used to separate possible luminescence contributions. The signals were detected by a Peltier cooled charge-coupled-devices (CCD) camera. The measured SHG signals were normalized by the squared laser intensity. The sample was positioned in a cryostat with a superconducting split-coil magnet. The temperature could be varied from 1.4 to $50 \mathrm{~K}$. Magnetic fields up to $B=10 \mathrm{~T}$ were applied in the Voigt geometry (see above). As already mentioned in the previous section the experiments were performed at normal light incidence with $\mathbf{k} \|[111]$. The experimental geometry is shown in Fig. 4, using the same angle notations as introduced above.

The EuTe and EuSe epilayers were grown by MBE on the (111) surface of cubic $\mathrm{BaF}_{2}$ substrates, and capped with a 40-nm-thick $\mathrm{BaF}_{2}$ protective layer. ${ }^{60,75} \mathrm{BaF}_{2}$ is transparent in the range of the fundamental and SHG wavelengths investigated here and does not contribute to the measured SHG signals. One sample had a $1 \mu \mathrm{m}$ thick EuTe layer and another one a $0.5 \mu \mathrm{m}$ thick EuSe layer. The high structural quality of these layers was confirmed by $\mathrm{x}$-ray analysis. 
While there is a considerable $6 \%$ lattice mismatch between EuTe and $\mathrm{BaF}_{2}$, the $1 \mu \mathrm{m}$ thick EuTe epilayer is fully relaxed to its bulk lattice constant, with a residual strain of only about $0.1 \%$ as checked by $\mathrm{x}$-ray diffraction. EuSe epilayer, on the other hand is practically lattice matched to $\mathrm{BaF}_{2}$ with a difference in lattice constant of only $0.08 \%$. The EuSe layer is therefore pseudomorphic to the $\mathrm{BaF}_{2}$ substrate and exhibits only a negligible in plane strain. Upon cooling to cryogenic temperatures, additional thermal strains may develop in the epilayers due to differences in the thermal expansion coefficient with respect to the substrate. However, for EuTe, EuSe, and $\mathrm{BaF}_{2}$ the thermal coefficients are very close to each other and therefore, such a thermal strain should be less that $0.2 \%$ even at liquid helium temperatures.

The epilayers used here with micrometer thicknesses can be regarded as bulk-like with physical properties similar, but superior, to those of bulk crystals. State of the art for $\mathrm{Eu} X$ technology is that MBE grown epilayers have much higher structural and optical quality compared to bulk single crystals. ${ }^{59,88}$ For higher order nonlinear optical effects the sample quality is a very important and often decisive factor. Requirements such as chemical stoichiometry and sample stability, structural and optical homogeneity, absence of impurities, partial SHG phase matching are easier fulfilled in MBE epilayers than in bulk crystals of EuX.

\section{EXPERIMENTAL RESULTS}

In this part, we present experimental results on SHG in EuTe and EuSe samples and discuss their dependencies on external magnetic field and temperature in the frame of a phenomenological approach.

\section{A. Second harmonic generation in EuTe}

Figure 6 shows SHG spectra of EuTe recorded at different magnetic fields at a temperature of $1.4 \mathrm{~K}$. The light wave vector in this experiment is parallel to the [111] normal vector of the EuTe surface. At zero magnetic field no SHG signal was detected in a wide temperature range from 1.4 to 100 $\mathrm{K}$ below and above $T_{N}$. From that we conclude that the symmetry reduction caused by the trigonal strain due to the mismatch to the substrate is very weak as it is not sufficient to break the inversion symmetry and induce detectable SHG intensity. As we had shown before, ${ }^{33,35}$ this is a favorable experimental situation to study nonlinear optical phenomena induced by external magnetic fields, as the induced signals are not screened by the typically strong crystallographic contributions. In magnetic fields above $3 \mathrm{~T}$ SHG signal appears in the vicinity of the band gap. Its spectral shape with a maximum at $2.4 \mathrm{eV}$ and a shoulder at $2.2 \mathrm{eV}$ is in good agreement with the reported EuTe absorption spectra, ${ }^{61,62}$ as shown below in Fig. 15(a).

The dependence of the SHG intensity integrated over the whole spectrum in the range from 2.1 to $2.5 \mathrm{eV}$ on the magnetic field strength is given in Fig. 7. The SHG intensity increases with growing magnetic field and saturates for $B$ $>7.5 \mathrm{~T}$. This behavior is in good qualitative agreement with the magnetization properties of EuTe. From Eq. (8) one can

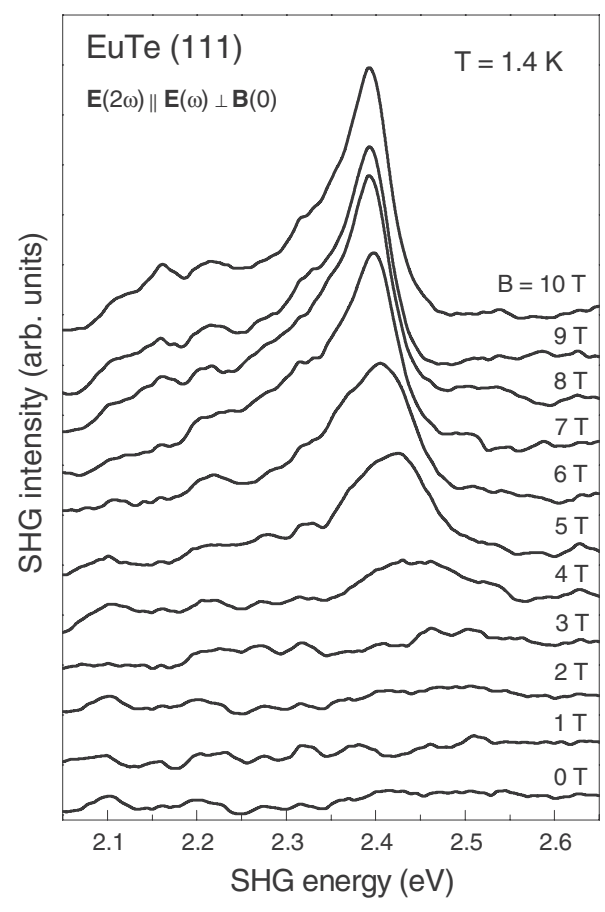

FIG. 6. SHG spectra of EuTe measured for different magnetic field strengths.

suggest that $I(2 \omega) \propto\left|\mathbf{P}^{I M D}(2 \omega)\right|^{2} \propto M^{2}(B)$, and indeed the normalized dependence of $M^{2}(B)$ plotted by the dashed line follows well the experimental data. These are also in reasonable agreement with the results of microscopic calculations shown by the solid line, which we will discuss in detail in Sec. VI B. It is evident from these results that the SHG mechanism in EuTe is controlled by the spin polarization of the $\mathrm{Eu}^{2+}$ ions induced by an external magnetic field.

The magnetization field dependence of EuTe below $T_{N}$ arises from a continuous transformation of the AFM ordering

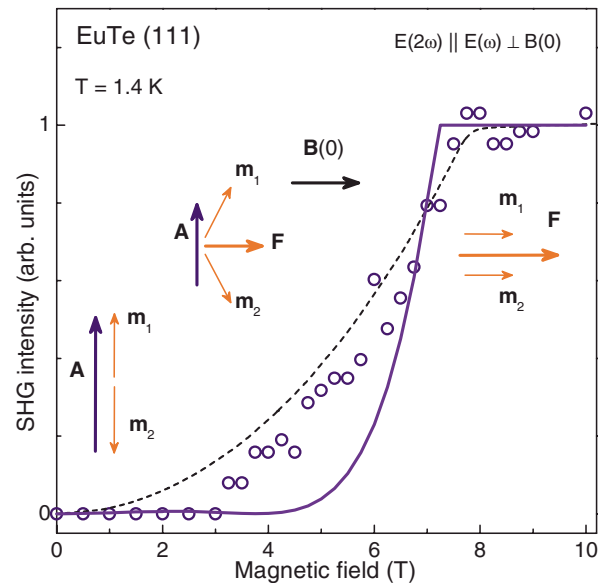

FIG. 7. (Color online) Integral SHG intensity as function of magnetic field. Experimental data are shown by circles. Dashed line gives the normalized $M^{2}(B)$ after Ref. 89 which is the basis for our phenomenological description. Solid line is result of the microscopic calculations described in Sec. VI A. Insets show schematically the reorientation of the magnetic sublattices and their transformation from AFM to FM alignment with increasing magnetic field. 


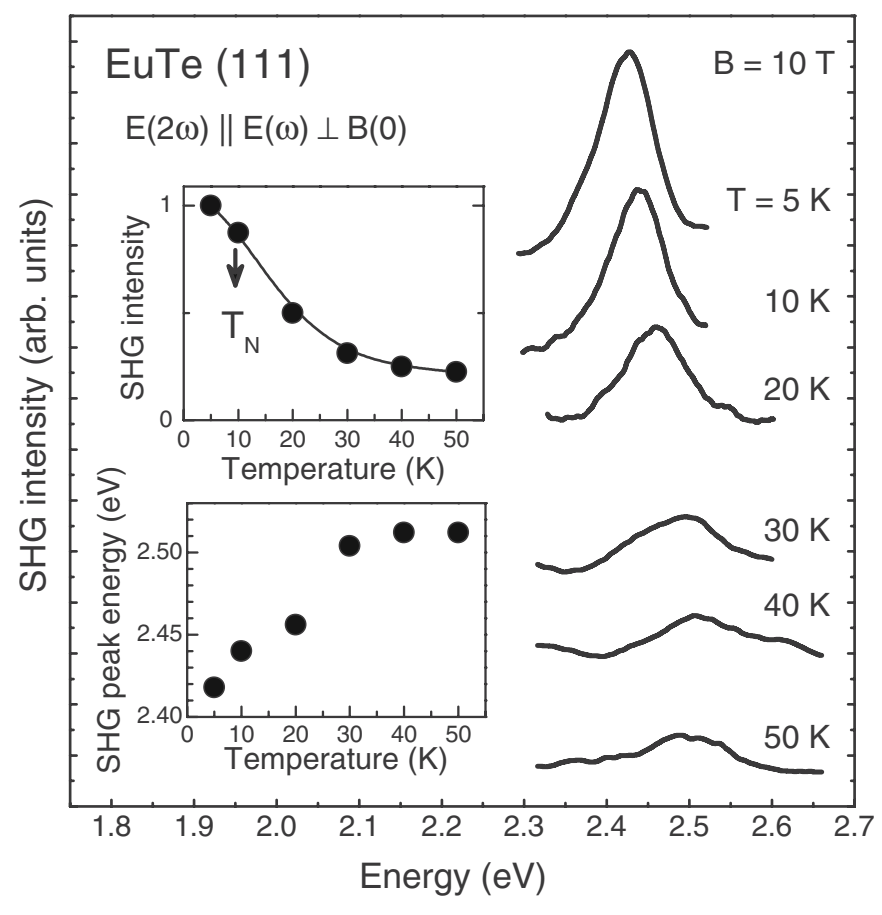

FIG. 8. SHG spectra of EuTe for different temperatures. The upper inset shows the SHG peak intensity as function of temperature. The solid line gives the normalized $M^{2}(T)$ after Ref. 89 . The lower inset shows the temperature shift of the peak energy.

at $B=0$ to a FM one above $B_{\text {sat }}=7.2$ T. This is shown schematically in the insets of Fig. 7. The AFM vector $\mathbf{A}$ $=\mathbf{m}_{1}-\mathbf{m}_{2}$ (for its definition see Sec. II C) does not induce any SHG signal, which qualitatively can be understood as follows: At $B=0$, the magnetic moments of two sublattices $\mathbf{m}_{1}$ and $\mathbf{m}_{2}$ are oriented antiferromagnetically. Each magnetic sublattice induces an SHG signal via a MD contribution according to Eq. (8) where $\mathbf{M}(0)$ should be substituted by $\mathbf{m}_{1}$ or $\mathbf{m}_{2}$. However, the destructive interference of signals from the oppositely oriented sublattices annihilates the SHG signal since the relevant nonlinear polarization is an odd function of $\mathbf{M}(0)$. With increasing magnetic field the AFM ordering is transformed into a FM one and the destructive interference is continuously reduced. In this case, $\mathbf{M}(0)$ in Eq. (8) should be associated with the ferromagnetic vector $\mathbf{F}=\mathbf{m}_{1}+\mathbf{m}_{2}$. Then the SHG signal intensity should increase with magnetic field as $I(2 \omega) \propto M^{2}(B)$, and reach saturation above $B_{\text {sat }}$ when the two sublattices have become oriented ferromagnetically and the magnetization has saturated as is experimentally observed (see Fig. 7).

This qualitative consideration can be extended to describe the temperature dependence of the SHG intensity. Above $T_{N}$ in the paramagnetic phase $\mathbf{M}(T)=\chi_{p}(T) \mathbf{B}$, where $\chi_{p}$ is the paramagnetic susceptibility. Therefore, SHG is also expected to be induced by an external magnetic field. The SHG intensity should decrease with growing temperature due to the decrease of the paramagnetic susceptibility.

SHG spectra measured for different temperatures from 5 to $50 \mathrm{~K}$ at a magnetic field of $B=10 \mathrm{~T}$ are shown in Fig. 8 . The SHG intensity continuously decreases with increasing temperature and vanishes above $50 \mathrm{~K}$. As one can see from the upper inset in Fig. 8 this decrease follows approximately

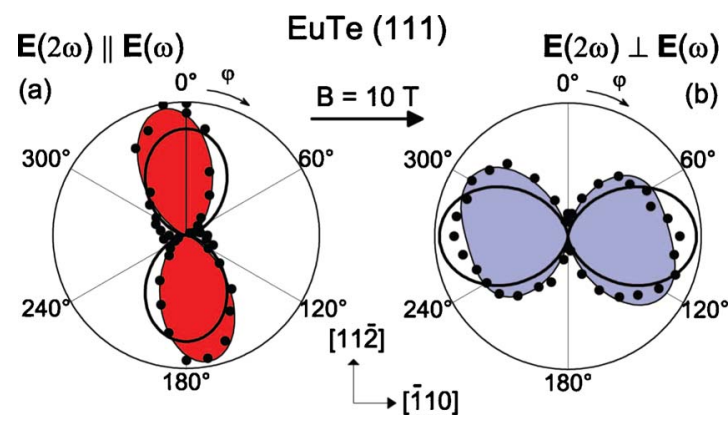

FIG. 9. (Color online) Polar plots of the experimental SHG intensity data (circles) at $2.4 \mathrm{eV}$ and $T=1.4 \mathrm{~K}$. Best fits based on Eqs. (12)-(16), taking into account the IMD only (solid lines) or alternatively, both the IMD and CMD (shaded areas).

the $M^{2}(T)$ dependence, ${ }^{89}$ as predicted by our qualitative considerations given above. A major part of the temperature range shown here covers the paramagnetic regime for EuTe, which has a $T_{N}=9.58 \mathrm{~K}$ above which the SHG signal is obviously related to the paramagnetic spin polarization.

The rotational anisotropies measured for an EuTe sample with simultaneous rotation of linear polarizers for the fundamental and SHG light are displayed in Fig. 9. Both for the parallel $\mathbf{E}(2 \omega) \| \mathbf{E}(\omega)$ and perpendicular $\mathbf{E}(2 \omega) \perp \mathbf{E}(\omega)$ configurations the SHG rotation patterns are twofold symmetric, having, however, slight distortions, which become more evident for the $\mathbf{E}(2 \omega) \perp \mathbf{E}(\omega)$ configuration. As it has been shown in Fig. 5 the twofold patterns correspond to the induced magnetic dipole nonlinear polarization $P^{I M D}$. The solid lines in Fig. 9 show the calculated SHG intensities according to Eqs. (12) and (13), which account only for IMD contributions. They are in satisfactory agreement with the experimental data points shown by circles.

The minor role of the CMD contribution for EuTe is confirmed by the magnetic field dependence of the SHG intensity which closely follows the dependence $I(2 \omega) \propto M^{2}$, as seen in Fig. 7. The CMD contribution is not detectable at low magnetic fields, but it affects the rotational anisotropy in strong magnetic fields causing slight distortions from the twofold diagrams shown by the solid line. We fit the experimental data in Fig. 9 with Eqs. (15) and (16) accounting for interference of the IMD and CMD contributions. The results are given by the shaded areas. We find from these fits that in the saturation regime of high-magnetic fields the ratio of polarizations $P^{I M D} / P^{C M D} \sim 7 / 1$, which after conversion into SHG intensities gives $I^{I M D}(2 \omega) / I^{C M D}(2 \omega) \sim 49 / 1$. It is, therefore, not surprising that the intensity related to the CMD contribution only is so weak that it cannot be detected at zero magnetic field.

\section{B. Second harmonic generation in EuSe}

The experimental manifestation of magnetic-field-induced SHG in EuSe is qualitatively similar to the one in EuTe. SHG spectra measured for the two configurations of parallel and crossed polarizations of the fundamental and generated light waves are shown in Fig. 10 in the spectral range 2.2$2.8 \mathrm{eV}$, i.e., in the vicinity of the optical band gap. SHG 


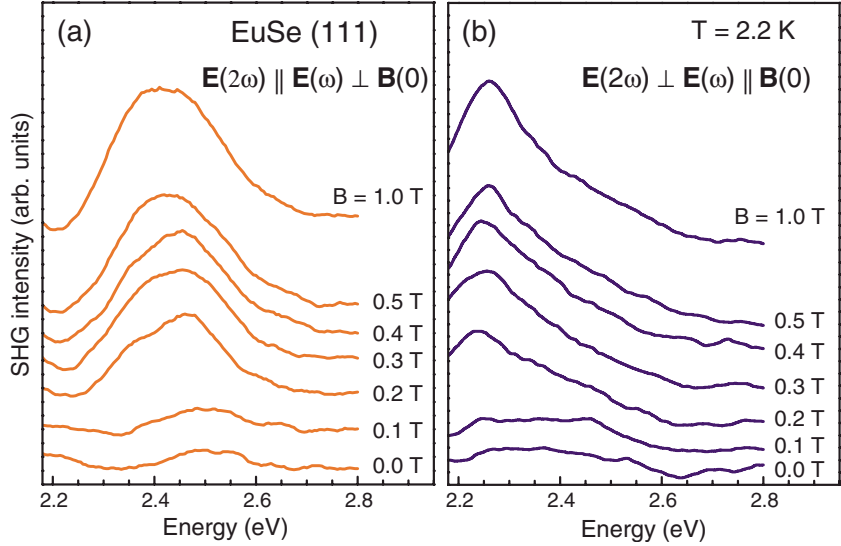

FIG. 10. (Color online) Magnetic-field-induced SHG spectra of EuSe measured at $2.2 \mathrm{~K}$ for different magnetic fields in two experimental geometries.

signal is practically absent at zero magnetic field, but shows up in finite field and increases with field strength. However, contrary to EuTe where the signal starts to be detectable for magnetic fields above $3 \mathrm{~T}$ and saturates above $7.5 \mathrm{~T}$ (Fig. 7), in EuSe signal is seen already at $0.1 \mathrm{~T}$ and saturates above $0.2 \mathrm{~T}$. In addition, the spectral shape of the signals depends on the experimental geometry: In panel (a) for $\mathbf{E}(2 \omega) \| \mathbf{E}(\omega) \perp \mathbf{B}(0)$ the broad peak at $B=1 \mathrm{~T}$ is centered at $2.45 \mathrm{eV}$, while in panel (b) for $\mathbf{E}(2 \omega) \perp \mathbf{E}(\omega) \| \mathbf{B}(0)$ the peak is at $2.25 \mathrm{eV}$. We have performed measurements in magnetic fields up to $10 \mathrm{~T}$ and found no pronounced variations in SHG signal intensities and spectral shapes in the range of 1-10 T.

The magnetic field dependence of the SHG intensity for the two experimental geometries is shown in Fig. 11. In both cases, the SHG intensity increases in a stepwise manner with growing field strength and shows two saturation regions: the first one between 0.01 and $0.2 \mathrm{~T}$ and the second one above $0.2 \mathrm{~T}$. These steps are in good agreement with the critical fields for the magnetic phase transitions in EuSe from AFM to FIM and FIM to FM, respectively. ${ }^{59}$ It is important to note, that this agreement gives us a clear proof that the measured SHG signal arises from the bulk of the sample and not

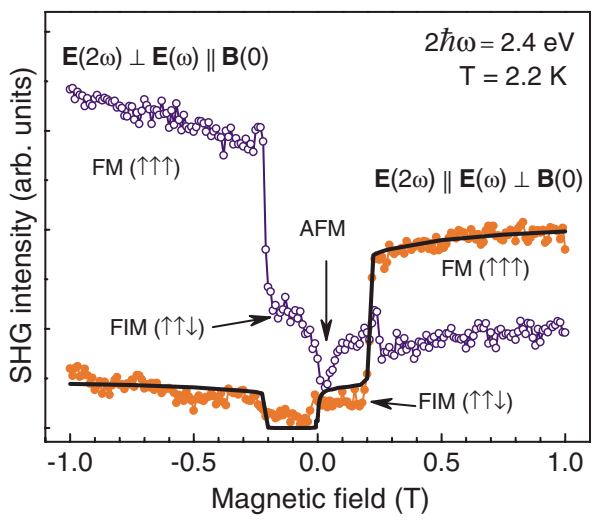

FIG. 11. (Color online) SHG intensity as function of magnetic field strength at an energy of $2.4 \mathrm{eV}$. The line gives normalized values calculated after $[a+b M(B)]^{2}$ with $b / a=4$ and $M(B)$ after Ref. 59. from its surface. ${ }^{11}$ The critical fields for magnetic phase transitions at the surface, and in particular in antiferromagnets, radically differ from those in bulk.

It is interesting that the magnetic field dependence of the SHG intensity shown in Fig. 11 is strongly asymmetric with respect to field inversion. E.g., for the $\mathbf{E}(2 \omega) \| \mathbf{E}(\omega) \perp \mathbf{B}(0)$ configuration the saturation level for the positive field direction is about four times larger than the one for negative fields. This behavior cannot be explained only by magneticfield-induced contributions to the SHG signals, e.g., given by Eq. (8), which should be symmetric with respect to the field inversion. It is clear that we have an additional contribution, which does not change its sign with field inversion. It is reasonable to suggest that this is the CMD contribution induced by a strain distortion and described by Eq. (7). The asymmetric dependencies in Fig. 11 result from interference of the IMD and CMD contributions, see Eq. (14).

To model the magnetic field dependence of the SHG signal in Fig. 11 taking into account the interference of the CMD and IMD contributions we use the function $I(2 \omega)$ $\propto[b M(B)+a]^{2}$ which has been derived from Eq. (14). Here the first term is proportional to $P^{I M D}$ and the constant $a$ to $P^{C M D}$. We suggest in this approach that in the studied field range $P^{C M D}$ is independent of magnetic field strength. A ratio of the constants $b / a=4$ has been obtained from the best fit of the rotational anisotropy patterns in Fig. 13, from which in the saturation regime a ratio $P^{I M D} / P^{C M D} \approx 4 / 1$ has been extracted as fitting parameter. The dependence $M(B)$ was taken from experimental data on the magnetization in EuSe reported in Ref. 59. The resulting fit, which includes only one scaling factor to adjust the intensity is shown by the bold solid line in Fig. 11. It reproduces well the asymmetry behavior of the field dependence confirming the validity of the used model.

The SHG spectra at different temperatures and the temperature dependence of the SHG intensity are shown in Fig. 12. Not only the intensity but also the spectral shape varies with temperature. At the lowest temperature of $1.4 \mathrm{~K}$ the peak intensity in the $\mathbf{E}(2 \omega) \perp \mathbf{E}(\omega) \| \mathbf{B}(0)$ configuration is about twice larger than the one in the $\mathbf{E}(2 \omega) \| \mathbf{E}(\omega) \perp \mathbf{B}(0)$ configuration, see panel (a). Also the double peak structure is seen for the $\mathbf{E}(2 \omega) \| \mathbf{E}(\omega) \perp \mathbf{B}(0)$ geometry. A temperature increase to $2.4 \mathrm{~K}$, panel (b), reduces the intensity difference between the two spectra and also the second peak becomes hardly visible as it is transformed in a high energy shoulder. Further temperature increase did not yield strong changes of the ratio of the amplitudes and the shape, while the total SHG intensity decreases as displayed in panel (d). We were not able to detect SHG signal for temperatures above $15 \mathrm{~K}$.

Figure 13 presents the rotational anisotropies for oppositely directed fields of $B=1$ and $-1 \mathrm{~T}$. Evidently the anisotropies strongly depend on the field direction and show apparently discrepancies from the pure induced magnetic dipole contribution $P^{I M D}$ shown by the solid lines. The discrepancies are especially obvious in panels (b) and (c), where the experimental patterns are rather quasi-fourfold than twofold as expected for the IMD contribution. Modeling according to Eqs. (15) and (16) with accounting for the interference of the IMD and CMD contributions gives a ratio of $P^{I M D} / P^{C M D}$ $=4$ as parameter for the best fit of the data, where the fit is 

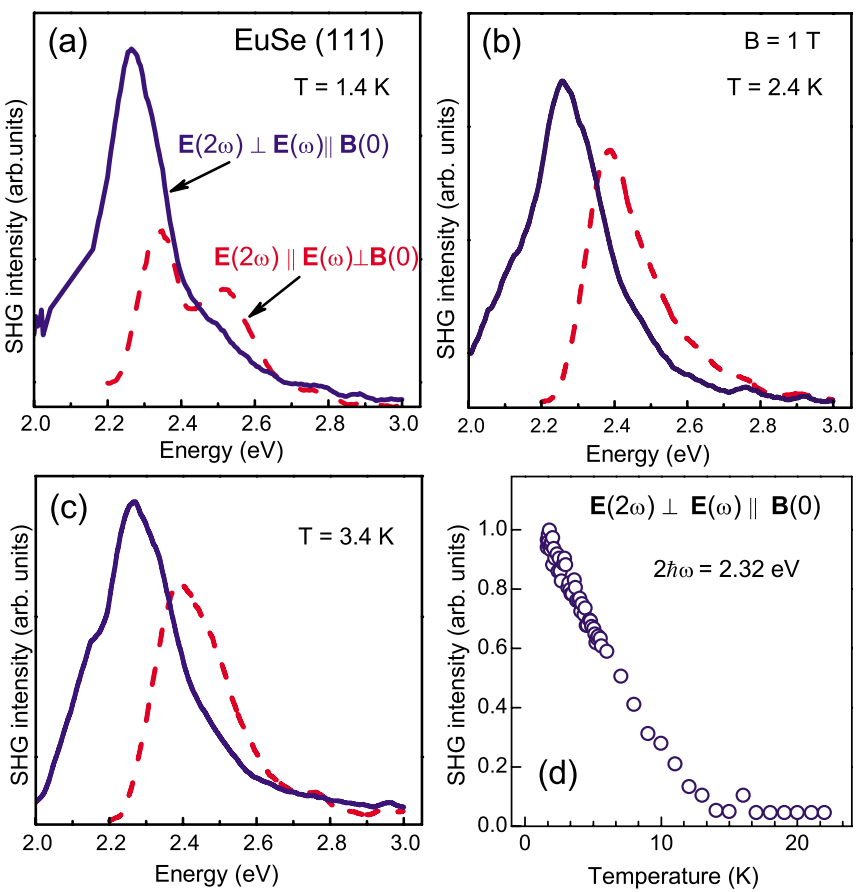

FIG. 12. (Color online) (a)-(c) SHG spectra of the EuSe sample for different temperatures measured at $B=1 \mathrm{~T}$ in two experimental geometries. (d) Peak intensity versus temperature.

represented as shaded areas in Fig. 13. One may also note that the rotational diagram shapes are similar to the ones obtained when the field inversion is accompanied by a change experimental configuration, compare panels (a), (d) and (b), (c) in Fig. 13.

Detailed results for transformation of the rotational anisotropy patterns for planar rotation of the EuSe sample with respect to the external field direction are presented in Fig. 14

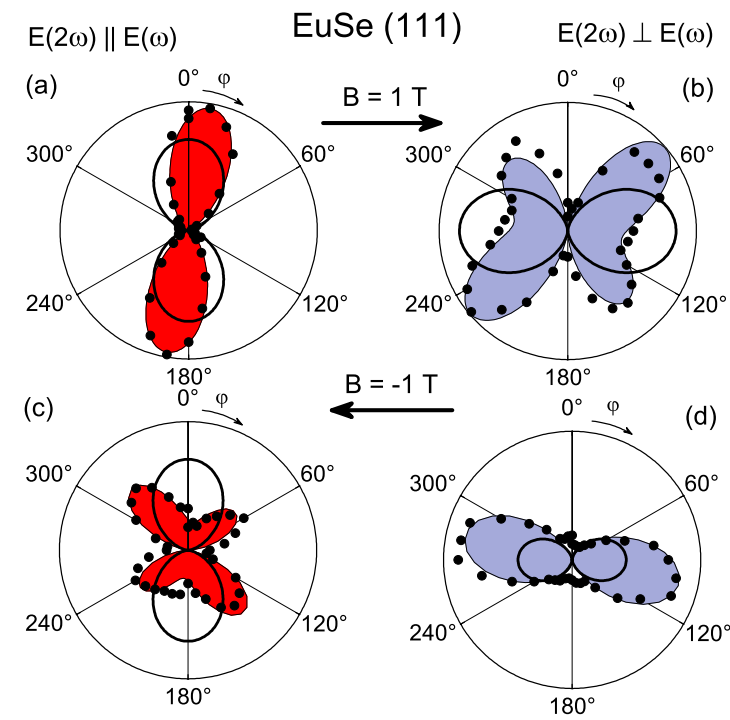

FIG. 13. (Color online) Polar plots of experimental SHG intensity data (dots) in EuSe measured at $2 \hbar \omega=2.4 \mathrm{eV}$ for opposite magnetic field directions. Best fits based on Eqs. (12)-(16), taking into account IMD (IMD and CMD) are shown by the solid lines (the shaded areas). for different angles $\alpha$ between the crystallographic [110] axis and the applied magnetic field direction. One can note few characteristic features for the pattern transformations:

(i) The rotational diagrams are invariant under rotations by $\alpha=120^{\circ}$. This can be seen, e.g., by comparison of panels (a) and (o) where we show here one period only.

(ii) Rotations by $60^{\circ}$ provide alternative transformations between twofold and fourfold patterns: panels (a), (i) and (o) for $\mathbf{E}(2 \omega) \| \mathbf{E}(\omega)$ and (b), (j) and (p) for $\mathbf{E}(2 \omega) \perp \mathbf{E}(\omega)$.

(iii) Rotations by $60^{\circ}$ degree are equivalent to a change of the experimental geometry and simultaneous rotation of the pattern by $90^{\circ}$, compare panels (a) with (j) and (b) with (i).

(iv) Sample rotations by $60^{\circ}$ lead to the same modifications as inversion of the magnetic field direction, compare panels (i) and (j) in Fig. 14 for $B=1 \mathrm{~T}$ and $\alpha=-69^{\circ}$ with panels (c) and (d) in Fig. 13 for $B=-1$ T and $\alpha=0^{\circ}$.

The SHG rotational anisotropy patterns in Fig. 14 can be explained on the basis of the IMD and CMD patterns depicted in Fig. 5. Let us demonstrate this for the $\mathbf{E}(2 \omega) \| \mathbf{E}(\omega)$ geometry corresponding to panels (a) and (b). The induced magnetic contribution has a twofold symmetry and is aligned perpendicular to the magnetic field direction, while the crystallographic contribution is sixfold and is linked to the crystallographic axis. Therefore, the CMD pattern is rotated with respect to the IMD when the angle $\alpha$ varies. This results in transformations of the SHG signal which is controlled by the interference of the IDM and CMD contributions. The validity of this approach is confirmed by the good quantitative agreement achieved by modeling of the experimental patterns, as shown by the shaded areas in Fig. 14.

\section{DISCUSSION}

\section{A. Microscopic model of SHG}

A microscopic model for magnetic-field-induced SHG processes in the magnetic semiconductors $\mathrm{Eu} X$ in the vicinity of the band gap has been proposed recently in Ref. 38. It is based on the energy level structure described in Sec. II B and allows one to calculate the SHG spectrum, the rotational anisotropy of the SHG signal, and the magnetic field dependence of the SHG intensity at $T=0 \mathrm{~K}$. In this Section we briefly describe the SHG model for $\mathrm{Eu} X$; for a detailed description refer to Ref. 38.

The SHG model explores two fundamental properties of the energy level scheme: (i) the energy spread of the excited states is much smaller than the band gap; (ii) all excited states have the same parity, which is opposite to the parity of the ground state. From the first property, it can be assumed that the dominant second order induced polarization will be strongly resonant when the photon energy is about $\hbar \omega$ $\sim E_{g} / 2$. Therefore, we may discard the anti-resonant contributions $^{1,2}$ to the induced second-order dipole moment. From the second property, the ED matrix elements between excited states vanish. The excited states may be connected through the MD operator, whereas their coupling by the EQ is much weaker. ${ }^{38}$ Consequently the complex amplitude of the second-order polarization vector component, which is parallel to the polarization vector (taken to be along the $x$ axis) of the exciting photons of energy $\hbar \omega$, is given by 


\section{EuSe (111)}
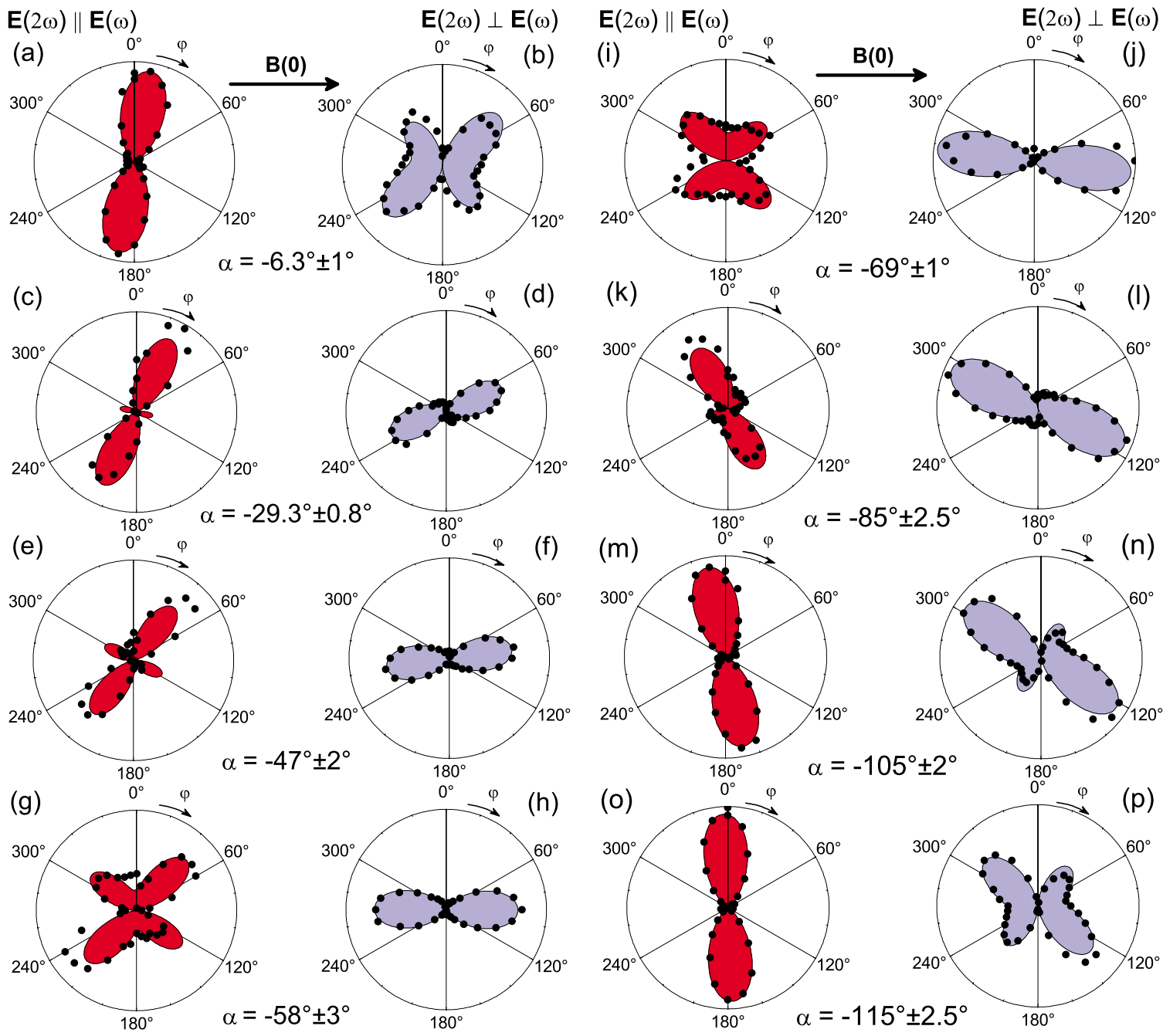

FIG. 14. (Color online) Polar plots of the SHG intensity data (the dots) in EuSe measured at $2.4 \mathrm{eV}$ for different angles $\alpha$ describing an in plane rotation of the sample, see Fig. 4. $\alpha=0$ corresponds to parallel orientation of the [110] axis and the magnetic field direction. Fits based on Eqs. (15) and (16), accounting for the interference of IMD and CMD contributions, are shown by the shaded areas.

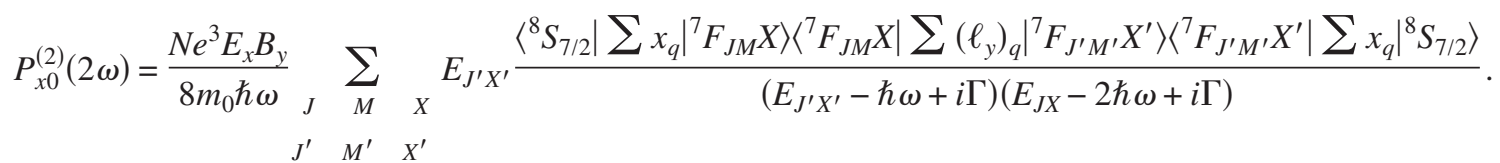

Here, $N=4 / a_{0}^{3}$ is the concentration of $\mathrm{Eu}^{2+}$ ions in the lattice, $a_{0}$ is the size of the fcc unit cell of $\mathrm{Eu} X, E_{x}$ and $B_{y}$ are the amplitudes of the electric and magnetic fields of the excitation laser, respectively, and $E_{J X}$ is given by Eq. (6). The relaxation between any excited state and the ground state, is described by the common parameter $\Gamma . x_{q}$ and $\left(\ell_{y}\right)_{q}$ represent the $x$ component of the position operator, and the $y$ component of the angular momentum, respectively, for the $q$-th electron $(q=1, \ldots, 7)$, and the sum is taken over all seven electrons involved in the electronic transitions. Equation (17) allows for an interpretation of the SHG process in terms of a simultaneous three-photon process. The initial seven electron ground state $4 f^{7}\left({ }^{8} S_{7 / 2}\right)$ is excited into a ${ }^{7} F_{J M} X$ virtual state through an ED induced by the fundamental incident light, which also induces a MD between virtual states ${ }^{7} F_{J M} X$ and ${ }^{7} F_{J^{\prime} M^{\prime}} X^{\prime}$. The electronic dipole between the ${ }^{7} F_{J^{\prime} M^{\prime}} X^{\prime}$ virtual state and the ground state $4 f^{7}\left({ }^{8} S_{7 / 2}\right)$ produces a photon resonant with the band-gap energy.

The SHG polarization given by Eq. (17) depends strongly on magnetic field, because the field strength and direction determine the orientation of the $\mathrm{Eu}^{2+}$ spins in the $\mathrm{EuX}$ lattice. ${ }^{62,82}$ This has immediate impact on the magnitude of the matrix elements in Eq. (17), and explains the sensitivity of the SHG intensity on magnetic field. In zero field, in EuTe and EuSe the lattice is antiferromagnetic, in which case the induced second-order polarization of the two sublattices can- 


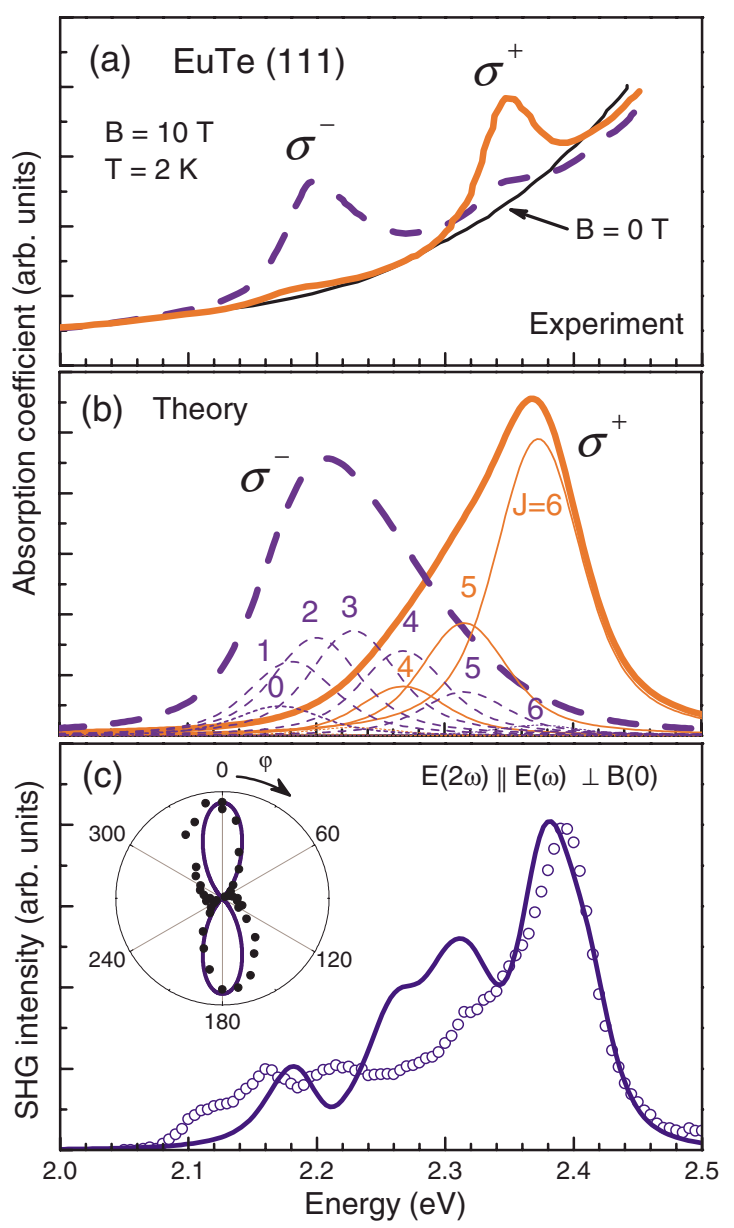

FIG. 15. (Color online) Comparison of experimental (Ref. 61) (a) and calculated (Ref. 62) (b) linear absorption spectra in EuTe at $B=10 \mathrm{~T}$. (c) Comparison of SHG spectra (line) calculated in the frame of the microscopic model (Ref. 38) and the experimental data (circles).

cel each other. When a magnetic field imposes ferromagnetic order, the total second-order polarization becomes nonzero.

SHG spectra were calculated using Eq. (17) for the same geometry as in experiment below, i.e., when the linearly polarized fundamental light is transmitted along the [111] crystal direction, and the magnetic field is applied perpendicular to the light propagation (Voigt geometry). The values of the input parameters used in the calculations are given in Table I. The only adjustable parameter was the relaxation rate $\Gamma$, which was fixed at $\Gamma=10 \mathrm{meV}$ for best agreement with the experimental data. The results of the theory are compared to the experimental data in Sec. V A (Fig. 7) and Sec. VI B (Fig. 15).

\section{B. Comparison of experimental and calculated SHG}

The experimental data presented in Sec. V show that SHG can be generated in centrosymmetric EuTe and EuSe near the band gap. The ED-SHG contribution is strictly forbidden in these materials and through studies involving magnetic field and temperature variations, in combination with the strongly anisotropic SHG response with respect to the incident- outgoing light polarizations we have proven it to be related to the MD susceptibility $\chi^{e e m m}$, see Eq. (8). A theoretical microscopical analysis of the SHG spectra based on the knowledge of the electronic structure was presented in Sec. VI A. Here, we compare the experimental data with the theoretical calculations.

In Fig. 15 we compare the experimental and theoretical near band gap absorption spectra [panels (a) and (b)] and the SHG spectrum of EuTe [panel (c)]. The calculations were done according to Sec. VI A. Contributions of different transitions to the total absorption intensity are shown in Fig. 15(b). It turns out that according to the selection rules two different sets within the seven transitions could separately be excited by $\sigma^{+}$and $\sigma^{-}$circularly polarized light. The broad SHG band measured at $B=10 \mathrm{~T}$ has a maximum at $2.4 \mathrm{eV}$ and a shoulder at $2.2 \mathrm{eV}$, see Fig. 15(c). This is in good agreement with the EuTe absorption spectra from Fig. 15(a). ${ }^{61,62}$ The calculated SHG spectrum shown by the solid line resembles closely the experimental SHG spectrum. The differences between the theoretical and experimental spectra can be related to neglecting the broadening of the resonances and to interference effects between magnetic and crystallographic contributions.

Interesting conclusions can be drawn from the field dependence of the SHG intensity. The case of EuSe is too complicated for an analysis due to the presence of several magnetic phases which transform into one another as the applied magnetic field strength is varied. In EuTe the applied magnetic field smoothly changes the antiferromagnetic configuration into the saturated ferromagnetic one. As shown in Fig. 7 SHG signal is not detected at zero field in the antiferromagnetic phase and becomes saturated in high fields. According to our phenomenological theory and taking into account only the MD process described by Eq. (8), one might expect that the SHG intensity must vary as the square of the magnetization as shown in Fig. 7 by the dashed line. In fact, this approach describes reasonably well the experimental SHG dependence on applied magnetic field, although the experimental SHG increase appears to be steeper than the growth of the magnetization squared. On the other hand, calculations in terms of the microscopic model predict a very weak reaction of the SHG intensity to the magnetic field below $4 \mathrm{~T}$ and a very steep rise at higher fields as shown by the solid line. We attribute the difference between the experimental data and the calculations to the fact that interference effects of the IMD and CMD contributions were neglected.

The IMD and CMD interference is manifested in the most distinct way in the SHG rotational anisotropy. The phenomenology of this feature has been discussed in Secs. III A and III B. If the IMD and CMD contributions are analyzed separately they show highly symmetric azimuthal angular dependencies as depicted in Fig. 5. However, when these two contributions interfere and the phase difference between them varies, the twofold and sixfold symmetry is broken and the symmetry planes of the anisotropy patterns are lost. The experimental data in Figs. 9, 11, 13, and 14 unambiguously prove the presence of interference effects. In most cases they can be quite well fitted using Eqs. (15) and (16). 


\section{CONCLUSIONS}

In conclusion, spin-induced SHG in the vicinity of the band gap has been found in the centrosymmetric magnetic semiconductors EuTe and EuSe. We show that the SHG is due to the bulk magnetic-dipole mechanism. The ferromagnetic component of the magnetic structure, or the spin polarization in the paramagnetic phase, in applied magnetic field are the main sources of the magnetic-dipole SHG. This is proven by SHG measurements at varying magnetic fields and temperatures. The magnetic-dipole SHG mechanism is expected to be several orders of magnitude weaker than the ED-SHG. Nevertheless, strong magnetic-dipole SHG signals have been observed and proven to be due to the unique properties of the magnetic semiconductors EuTe and EuSe possessing giant magneto-optical properties around the band gap formed by optical transitions involving $4 f$ and $5 d$ electronic orbitals of the magnetic $\mathrm{Eu}^{2+}$ ions.

Nonreciprocal effects appear, in particular in EuSe, which we explain by the interference of the crystallographic and spin-induced contributions. A microscopic theory of magnetic-dipole SHG has been developed recently in Ref. 38 which we have briefly discussed in this paper and compared it with our experimental data. The theory is based on the electronic band structure and its variations in magnetic field. Experimental SHG spectra and their variations with magnetic field and temperature are described reasonably well. In the $\mathrm{Eu} X$ compounds a new type of MD nonlinearity appears in external magnetic field. It can be considered as counterpart to the nonlinearity arising from electric field application to centrosymmetric media, which breaks space inversion symmetry and makes the electric-dipole SHG processes allowed. This type of spin-induced nonlinear susceptibility opens new opportunities for nonlinear optical spectroscopic studies of centrosymmetric bulk materials, thin films, and artificial structures.

\section{ACKNOWLEDGMENTS}

This work was supported by the Deutsche Forschungsgemeinschaft (Grant No. YA65/4-1); the Russian Foundation for Basic Research, Russian Academy Programs on Spintronics, Physics of Nanostructures, Quantum Physics of Condensed Matter; FoNE of the European Science Foundation; the Brazilian Agencies FAPESP and CNPq; the Deutscher Akademischer Austausch Dienst (DAAD), and the FWF (I80-N20, Vienna, Austria).
${ }^{1}$ Y. R. Shen, The Principles of Nonlinear Optics (Wiley, New York, 1984).

${ }^{2}$ R. W. Boyd, Nonlinear Optics (Academic Press, San Diego, 1993).

${ }^{3}$ M. Fiebig, V. V. Pavlov, and R. V. Pisarev, J. Opt. Soc. Am. B 22, 96 (2005).

${ }^{4}$ T. F. Heinz, C. K. Chen, D. Ricard, and Y. R. Shen, Phys. Rev. Lett. 48, 478 (1982).

${ }^{5}$ P. Guyot-Sionnest, W. Chen, and Y. R. Shen, Phys. Rev. B 33, 8254 (1986)

${ }^{6}$ P.-F. Brevet, Surface Second Harmonic Generation (Presses polytechniques et universitaires romandes, 1997).

${ }^{7}$ A. Kirilyuk, J. Phys. D 35, R189 (2002).

${ }^{8}$ A. Kirilyuk and T. Rasing, J. Opt. Soc. Am. B 22, 148 (2005).

${ }^{9}$ M. Fiebig, D. Fröhlich, Th. Lottermoser, V. V. Pavlov, R. V. Pisarev, and H.-J. Weber, Phys. Rev. Lett. 87, 137202 (2001).

${ }^{10}$ C. Li, G. Lefkidis, and W. Hübner, arXiv:0811.4042 (unpublished).

${ }^{11}$ J. E. Sipe, V. Mizrahi, and G. I. Stegeman, Phys. Rev. B 35, 9091 (1987).

${ }^{12}$ P. Guyot-Sionnest and Y. R. Shen, Phys. Rev. B 35, 4420 (1987).

${ }^{13}$ H. Hoshi, T. Yamada, K. Ishikawa, H. Takezoe, and A. Fukuda, J. Chem. Phys. 107, 1687 (1997).

${ }^{14}$ B. Koopmans, A.-M. Janner, H. T. Jonkman, G. A. Sawatzky, and F. van der Woude, Phys. Rev. Lett. 71, 3569 (1993).

${ }^{15}$ D. Wilk, D. Johannsmann, C. Stanners, and Y. R. Shen, Phys. Rev. B 51, 10057 (1995).

${ }^{16}$ T. Ishihara, K. Koshino, and H. Nakashima, Phys. Rev. Lett. 91, 253901 (2003).

${ }^{17}$ M. W. Klein, C. Enkrich, M. Wegener, and S. Linden, Science 313, 502 (2006).

${ }^{18}$ A. Q. Jiang, J. F. Scott, H. Lu, and Z. Chen, J. Appl. Phys. 93,
1180 (2003).

${ }^{19}$ M. Fiebig, D. Fröhlich, B. B. Krichevtsov, and R. V. Pisarev, Phys. Rev. Lett. 73, 2127 (1994).

${ }^{20}$ R. W. Terhune, P. D. Maker, and C. M. Savage, Phys. Rev. Lett. 8, 404 (1962).

${ }^{21}$ R. V. Pisarev, B. B. Krichevtsov, V. N. Gridnev, V. P. Klin, D. Fröhlich, and Ch. Pahlke-Learch, J. Phys.: Condens. Matter 5, 8621 (1993).

${ }^{22}$ V. V. Pavlov, R. V. Pisarev, A. Kirilyuk, and Th. Rasing, Phys. Rev. Lett. 78, 2004 (1997).

${ }^{23}$ V. N. Gridnev, V. V. Pavlov, R. V. Pisarev, A. Kirilyuk, and Th. Rasing, Phys. Rev. B 63, 184407 (2001).

${ }^{24}$ K. J. Choi, M. Biegalski, Y. L. Li, A. Sharan, J. Schubert, R. Uecker, P. Reiche, Y. B. Chen, X. Q. Pan, V. Gopalan, L. Q. Chen, D. G. Schlom, and C. B. Eom, Science 306, 1005 (2004).

${ }^{25}$ J. Reif, J. C. Zink, C. M. Schneider, and J. Kirschner, Phys. Rev. Lett. 67, 2878 (1991).

${ }^{26}$ M. Y. Shen, S. Koyama, M. Saito, T. Goto, and N. Kuroda, Phys. Rev. B 53, 13477 (1996).

${ }^{27}$ H. P. Wagner, M. Kühnelt, W. Langbein, and J. M. Hvam, Phys. Rev. B 58, 10494 (1998).

${ }^{28}$ S. Bergfeld and W. Daum, Phys. Rev. Lett. 90, 036801 (2003).

${ }^{29}$ K. L. Vodopyanov, G. M. H. Knippels, A. F. G. van der Meer, J. P. Maffetone, and I. Zwieback, Opt. Commun. 202, 205 (2002).

${ }^{30}$ K. L. Vodopyanov, F. Ganikhanov, J. P. Maffetone, I. Zwieback, and W. Ruderman, Opt. Lett. 25, 841 (2000).

${ }^{31}$ G. A. Medvedkin and V. G. Voevodin, J. Opt. Soc. Am. B 22, 1884 (2005).

${ }^{32}$ A. Godard, C. R. Phys. 8, 1100 (2007).

${ }^{33}$ V. V. Pavlov, A. M. Kalashnikova, R. V. Pisarev, I. Sänger, D. R. Yakovlev, and M. Bayer, Phys. Rev. Lett. 94, 157404 (2005).

${ }^{34}$ I. Sänger, D. R. Yakovlev, B. Kaminski, R. V. Pisarev, V. V. Pavlov, and M. Bayer, Phys. Rev. B 74, 165208 (2006). 
${ }^{35}$ I. Sänger, D. R. Yakovlev, R. V. Pisarev, V. V. Pavlov, M. Bayer, G. Karczewski, T. Wojtowicz, and J. Kossut, Phys. Rev. Lett. 96, 117211 (2006).

${ }^{36}$ I. Sänger, B. Kaminski, D. R. Yakovlev, R. V. Pisarev, M. Bayer, G. Karczewski, T. Wojtowicz, and J. Kossut, Phys. Rev. B 74, 235217 (2006).

${ }^{37}$ B. Kaminski, M. Lafrentz, R. V. Pisarev, D. R. Yakovlev, V. V. Pavlov, V. A. Lukoshkin, A. B. Henriques, G. Springholz, G. Bauer, E. Abramof, P. H. O. Rappl, and M. Bayer, Phys. Rev. Lett. 103, 057203 (2009).

${ }^{38}$ A. B. Henriques, E. Abramof, and P. H. O. Rappl, Phys. Rev. B 80, 245206 (2009).

${ }^{39}$ P. G. Steeneken, L. H. Tjeng, I. Elfimov, G. A. Sawatzky, G. Ghiringhelli, N. B. Brookes, and D.-J. Huang, Phys. Rev. Lett. 88, 047201 (2002).

${ }^{40}$ T. S. Santos and J. S. Moodera, Phys. Rev. B 69, 241203(R) (2004).

${ }^{41}$ T. S. Santos, J. S. Moodera, K. V. Raman, E. Negusse, J. Holroyd, J. Dvorak, M. Liberati, Y. U. Idzerda, and E. Arenholz, Phys. Rev. Lett. 101, 147201 (2008).

${ }^{42}$ G.-X. Miao, M. Müller, and J. S. Moodera, Phys. Rev. Lett. 102, 076601 (2009).

${ }^{43}$ G. Springholz, A. Raab, R. T. Lechner, and V. Holy, Appl. Phys. Lett. 79, 1225 (2001).

${ }^{44}$ J. S. Moodera, X. Hao, G. A. Gibson, and R. Meservey, Phys. Rev. Lett. 61, 637 (1988).

${ }^{45}$ X. Hao, J. S. Moodera, and R. Meservey, Phys. Rev. B 42, 8235 (1990).

${ }^{46}$ A. T. Filip, P. LeClair, C. J. P. Smits, J. T. Kohlhepp, H. J. M. Swagten, B. Koopmans, and W. J. M. de Jonge, Appl. Phys. Lett. 81, 1815 (2002).

${ }^{47}$ P. LeClair, J. K. Ha, H. J. M. Swagten, J. T. Kohlhepp, C. H. V. de Vin, and W. J. M. de Jonge, Appl. Phys. Lett. 80, 625 (2002).

${ }^{48}$ C. J. P. Smits, A. T. Filip, J. T. Kohlhepp, H. J. M. Swagten, B. Koopmans, and W. J. M. de Jonge, J. Appl. Phys. 95, 7405 (2004).

${ }^{49}$ J. Trbovic, C. Ren, P. Xiong, and S. von Molnar, Appl. Phys. Lett. 87, 082101 (2005).

${ }^{50}$ C. Ren, J. Trbovic, R. L. Kallaher, J. G. Braden, J. S. Parker, S. V. von Molnar, and P. Xiong, Phys. Rev. B 75, 205208 (2007).

${ }^{51}$ J. S. Moodera, R. Meservey, and X. Hao, Phys. Rev. Lett. 70, 853 (1993).

${ }^{52}$ D. D. Awschalom, D. Loss, and N. Samarth, Semiconductor Spintronics and Quantum Computation (Springer-Verlag, Berlin, 2002).

${ }^{53}$ D. Bouwmeester, A. Ekert, and A. Zeilinger, The Physics of Quantum Information (Springer-Verlag, Berlin, 2000).

${ }^{54}$ M. R. Koblischka and R. J. Wijngaarden, Supercond. Sci. Technol. 8, 199 (1995).

${ }^{55}$ J. Lettieri, V. Vaithyanathan, S. K. Eah, J. Stephens, V. Sih, D. D. Awschalom, J. Levy, and D. G. Schlom, Appl. Phys. Lett. 83, 975 (2003).

${ }^{56}$ A. Schmehl, V. Vaithyanathan, A. Herrnberger, S. Thiel, C. Richter, M. Liberati, T. Heeg, M. Röckerath, L. F. Kourkoutis, S. Mühlbauer, P. Böni, D. A. Muller, Y. Barash, J. Schubert, Y. Idzerda, J. Mannhart, and D. G. Schlom, Nature Mater. 6, 882 (2007).

${ }^{57}$ A. Mauger and C. Godart, Phys. Rep. 141, 51 (1986).

${ }^{58} \mathrm{P}$. Wachter, in Handbook on the Physics and Chemistry of Rare Earths, edited by K. A. Gschneider and L. R. Eyring (North
Holland, Amsterdam, 1979), Vol. 11, p. 507.

${ }^{59}$ R. T. Lechner, G. Springholz, T. U. Schülli, J. Stangl, T. Schwarzl, and G. Bauer, Phys. Rev. Lett. 94, 157201 (2005).

${ }^{60}$ L. K. Hanamoto, A. B. Henriques, N. F. Oliveira, Jr., P. Rappl, E. Abramof, and Y. Ueta, J. Phys.: Condens. Matter 16, 5597 (2004).

${ }^{61}$ A. B. Henriques, A. Wierts, M. A. Manfrini, G. Springholz, P. H. O. Rappl, E. Abramof, and A. Y. Ueta, Phys. Rev. B 72, 155337 (2005).

${ }^{62}$ A. B. Henriques, M. A. Manfrini, P. H. O. Rappl, and E. Abramof, Phys. Rev. B 77, 035204 (2008).

${ }^{63}$ T. Kasuya, Crit. Rev. Solid State Mater. Sci. 3, 131 (1972).

${ }^{64}$ T. Kasuya, IBM J. Res. Dev. 14, 214 (1970).

${ }^{65}$ D. E. Eastman, F. Holtzberg, and S. Methfessel, Phys. Rev. Lett. 23, 226 (1969).

${ }^{66}$ M. J. Freiser, S. Methfessel, and F. Holtzberg, J. Appl. Phys. 39, 900 (1968).

${ }^{67}$ J. Feinleib, W. J. Scouler, J. O. Dimmock, J. Hanus, T. B. Read, and C. R. Pidegeon, Phys. Rev. Lett. 22, 1385 (1969).

${ }^{68}$ J. Feinleib and C. R. Pidegeon, Phys. Rev. Lett. 23, 1391 (1969).

${ }^{69}$ G. Bauer, H. Pascher, and W. Zawadzki, Semicond. Sci. Technol. 7, 703 (1992).

${ }^{70}$ J. O. Dimmock, J. Hanus, and J. Feinleib, J. Appl. Phys. 41, 1088 (1970).

${ }^{71}$ J. O. Dimmock, IBM J. Res. Dev. 14, 301 (1970).

${ }^{72}$ G. Güntherodt, P. Wachter, and D. M. Imboden, Phys. Kondens. Mater. 12, 292 (1971).

${ }^{73}$ G. Güntherodt, Z. Phys. B: Condens. Matter 18, 37 (1974).

${ }^{74}$ R. Kirchschlager, W. Heiss, R. T. Lechner, G. Bauer, and G. Springholz, Appl. Phys. Lett. 85, 67 (2004).

${ }^{75}$ W. Heiss, G. Prechtl, and G. Springholz, Phys. Rev. B 63, 165323 (2001).

${ }^{76}$ J. Ferré, J. Phys. France 35, 781 (1974).

${ }^{77}$ J. Schoenes and P. Wachter, IEEE Trans. Magn. 12, 81 (1976).

${ }^{78}$ G. Busch, J. Schoenes, and P. Wachter, Solid State Commun. 8, 1841 (1970).

${ }^{79}$ J. Schoenes and P. Wachter, Physica B\&C 86-88, 125 (1977).

${ }^{80}$ M. Suekane, G. Kido, N. Miura, and S. Chikazumi, J. Magn. Magn. Mater. 31-34, 589 (1983).

${ }^{81}$ H. Hori, R. Akimoto, M. Kobayashi, S. Miyamoto, M. Furusawa, N. Kreines, A. Yamagishi, and M. Date, Physica B 201, 438 (1994).

${ }^{82}$ A. B. Henriques, G. D. Galgano, B. Diaz, P. H. O. Rappl, and E. Abramof, J. Phys.: Condens. Matter 19, 406234 (2007).

${ }^{83}$ A. B. Henriques, G. D. Galgano, and E. Abramof, J. Phys.: Condens. Matter 20, 255209 (2008).

${ }^{84}$ A. B. Henriques, L. K. Hanamoto, E. Ter Haar, E. Abramof, A. Y. Ueta, and P. H. O. Rappl, Int. J. Mod. Phys. B 18, 3813 (2004).

${ }^{85}$ G. F. Koster, J. O. Dimmock, R. G. Wheeler, and H. Statz, Properties of the Thirty-Two Point Groups (M.I.T. Press, Cambridge, MA, USA, 1963).

${ }^{86}$ P. S. Pershan, Phys. Rev. 130, 919 (1963).

${ }^{87}$ R. R. Birss, Symmetry and Magnetism (North Holland, Amsterdam, 1967).

${ }^{88}$ B. Diaz, E. Granado, E. Abramof, P. H. O. Rappl, V. A. Chitta, and A. B. Henriques, Phys. Rev. B 78, 134423 (2008).

${ }^{89}$ N. F. Oliveira, S. Foner, Y. Shapira, and T. B. Reed, Phys. Rev. B 5, 2634 (1972). 